\title{
ABSTRACTS \\ ASIM TCSE 2012 \\ Vienna
}

S. Tauböck, F. Breitenecker (eds).

\section{ASIM Workshop \\ Trends in Computational \\ Science \\ and Engineering}

February 13-14, 2012

Vienna University of Technology, Austria

\section{Programme}

ISBN print 978-3-901608-37-7

ISBN ebook 978-3-901608-05-6, DOI 10.11128/arep.37

ARGESIM Report no. 37 - ASIM Mitteilung AM 139 

ดับ는

ARGESIM

TWU 

ARGESIM Report no. 37 - ASIM Mitteilung AM 139

S. Tauböck, F. Breitenecker (eds).

\section{ABSTRACTS \\ ASIM TCSE 2012}

Vienna

\section{ASIM Workshop \\ Trends in Computational Science and Engineering}

February 13-14, 2012

Vienna University of Technology, Austria

ISBN print: 978-3-901608-37-7

ISBN ebook: 978-3-901608-05-6, DOI: 10.11128/arep.37

ARGESIM/ASIM, TU Vienna, 2012 
Published by ARGESIM and ASIM, German Simulation Society,

Div. of GI - German Society for Informatics / Informatics and Life Sciences

\section{Series Editor:}

Felix Breitenecker (ARGESIM / ASIM)

Div. Mathematical Modelling and Simulation,

Vienna University of Technology

Wiedner Hauptstrasse 8 - 10, 1040 Vienna, Austria

Tel: +43-1-58801-10115, Fax: +43-1-58801-10199

Email: Felix.Breitenecker@tuwien.ac.at

\section{ARGESIM Report no. 37 - ASIM Mitteilung AM 139}

Titel: Abstracts ASIM TCSE 2012 Vienna

Editors: $\quad$ Shabnam Tauböck, Felix Breitenecker

Div. Mathematical Modelling and Simulation,

Vienna University of Technology

Wiedner Hauptstrasse 8 - 10, 1040 Vienna, Austria

Email: Felix.Breitenecker@tuwien.ac.at

ISBN print 978-3-901608-37-7

ISBN ebook 978-3-901608-05-6, DOI: 10.11128/arep.37

Das Werk ist urheberrechtlich geschützt. Die dadurch begründeten Rechte, insbesondere die der Übersetzung, des Nachdrucks, der Entnahme von Abbildungen, der Funksendung, der Wiedergabe auf photomechanischem oder ähnlichem Weg und der Speicherung in Datenverarbeitungsanlagen bleiben, auch bei nur auszugsweiser Verwertung, vorbehalten.

(C) by ARGESIM / ASIM, Wien, 2012

ARGE Simulation News (ARGESIM)

c/o F. Breitenecker, Div. Mathematical Modelling and Simulation

Vienna Univ. of Technology

Wiedner Hauptstrasse 8-10, A-1040 Vienna, Austria

Tel.: +43-1-58801-10115, Fax: +43-1-58801-10199

Email: info@argesim.org; WWW: http://www.argesim.org 


\section{Content}

Content.

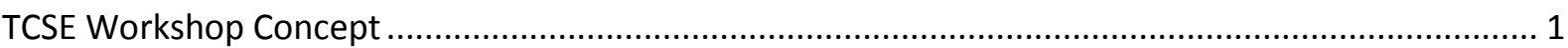

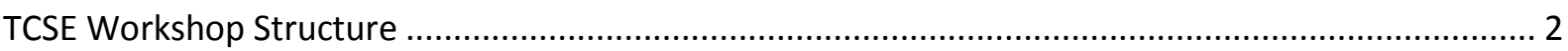

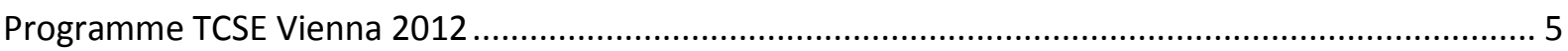

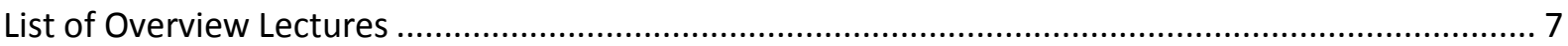

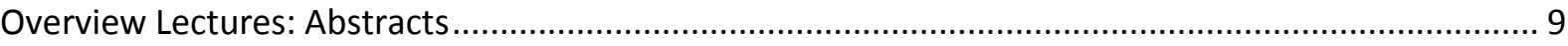

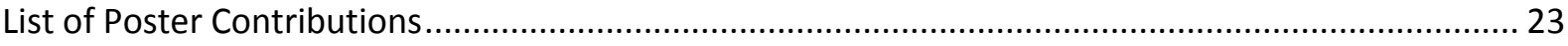

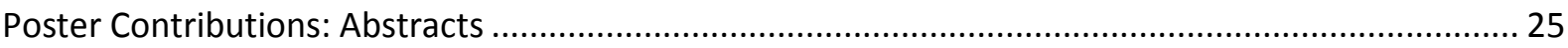

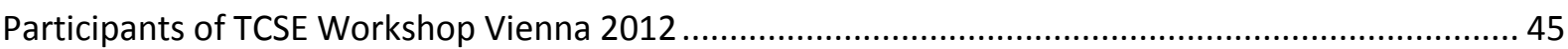

\section{TCSE Workshop Concept}

Modelling and simulation of complex systems from many fields of application requires a wide spectrum of methods, algorithms, and tools that cannot be surveyed even by experts. For many problems different solution approaches exist and the choice of a suitable method is usually determined by the modeller's experience and preferences. Direct comparisons of methods in practical applications are not commonly found in literature. The workshop series Trends in Computational Science - TCSE tries to fill this void.

The series has been initiated in 2004 by the ASIM working group Foundations and Methods in Modelling and Simulation (ASIM - German Society for Modelling and Simulation; www.asim-gi.org), the workshops are organized by this ASIM group and by the Trends-in-CSE group (www.trends-in.cse.de).

After Siegen (2004), Wuppertal (2005), Munich (2006), Aachen (2007), Erlangen (2008), Stuttgart (2009), Jülich (2010) and Munich (2011), the Vienna ASIM TCSE workshop is the ninth in this series.

The program aims at bridging the gap between basic algorithmic knowledge from different disciplines and practical applications in various fields of research.

Consequently, the workshop is focused on education for experts and on broadening the methodological knowledge in particular of $\mathrm{PhD}$ students and advanced master students from computational sciences as well as engineering sciences and related fields.

Overview presentations are given on a high but not over-specialized level to achieve a cross fertilization between the different modelling and simulation disciplines. For this reason, sufficient time is reserved for scientific discussions, cross linking of topics and networking of scientists. 


\section{TCSE Workshop Structure}

The TCSE workshop is particularly addressing advanced students and PhD candidates and is based on two scientific pillars:

- Overview Talks on trends in CSE

- Poster Session of PhD and Master Thesis projects in CSE and Modelling and Simulation

\section{Overview Talks on Trends in CSE}

At the workshop, overview talks on selected topics will be given by experts from the modelling and simulation community and from the computational science and engineering community.

The speakers will present methods, tools, and applications from various fields, such as multiscale methods, distributed and high performance computing, optimization and parameter estimation, digital image analysis, scientific workflows, modelling and simulation of technical and biological systems, distributed simulation, and cyber-physical systems.

\section{Poster Session on CSE PhD and Master Projects}

The TCSE workshop in particular addresses advanced students and PhD candidates who are encouraged to present their current work in a poster session. This session is given special emphasis in the workshop program: students can discuss their work and exchange ideas with experts from many fields and build up their own scientific networks.

The Poster Session traditionally starts in a lecture room with the Poster Rodeo, where each poster author gives a 3-minute summary on his work. After the Poster Rodeo participants are invited to proceed to the Poster Exhibition Area, where poster authors give detailed information on their work in front of their poster. Also in this session, sufficient time is reserved for discussions, cross linking of topics, and networking of scientists. The best posters will be selected by a jury and awarded with a prize.

\section{Organisers}

The workshop series is organised by ASIM, the German Society for Modelling and Simulation (www.asim-gi.org) and by the Trends-in-CSE group (www.trends-in.cse.de).

The local organiser of the ASIM TCSE 2012 workshop is ASC / TU Vienna:

Inst. for Analysis and Scientific Computing (ASC / TU Vienna)

Vienna University of Technology

Mathematical Modelling Group

Wiedner Hauptstrasse 8-10, 1040 Vienna, Austria 
The workshop is co-sponsored by

DWH Simulation Services

AIT Austrian Institute of Technology $\mathrm{GmbH}$, Health and Environment Department, Biomedical Systems

AMC TU Vienna - ARGESIM Master College on Modelling and Simulation

\section{Scientific Committee}

Prof. Dr. Felix Breitenecker, Dr. Shabnam Tauböck

Inst. for Analysis and Scientific Computing (ASC / TU Vienna), Vienna University of Technology

\section{Organizing committee}

Dr. Shabnam Tauböck, Brigitte Ecker

Inst. for Analysis and Scientific Computing (ASC / TU Vienna), Vienna University of Technology Barbara Glock

DWH Simulation Services / ASC

Bernhard Hametner, Xenia Descovich

AlT Austrian Institute of Technology $\mathrm{GmbH}$, Health and Environment Department, Biomedical Systems 


\section{Programme TCSE Vienna 2012}

\begin{tabular}{|c|c|}
\hline \multicolumn{2}{|c|}{$\begin{array}{c}\text { Monday } \\
\text { Feb. 13, } 2012 \\
\end{array}$} \\
\hline $13: 00-14: 00$ & $\begin{array}{l}\text { Registration } \\
\text { conference office }\end{array}$ \\
\hline $14: 00-16: 30$ & $\begin{array}{c}\text { Overview Talks } \\
\text { FH8 (Nöbauer), } 2^{\text {nd }} \text { floor, yellow area }\end{array}$ \\
\hline $16: 30-17: 00$ & $\begin{array}{l}\text { Coffee Break } \\
\text { yellow area }\end{array}$ \\
\hline $17: 00-19: 30$ & $\begin{array}{c}\text { Overview Talks } \\
\text { FH8 (Nöbauer), } 2^{\text {nd }} \text { floor, yellow area }\end{array}$ \\
\hline $19: 30$ & $\begin{array}{c}\text { Cider and Applejuice Tasting } \\
1^{\text {st }} \text { floor, red area }\end{array}$ \\
\hline
\end{tabular}

\section{Tuesday}

Feb. 14, 2012

9:00 - 11:00

Overview Talks

FH8 (Nöbauer), $2^{\text {nd }}$ floor, yellow area

$11: 00-11: 15$

Coffee Break yellow area

$11: 15-12: 00$

Poster Rodeo

FH8 (Nöbauer), $2^{\text {nd }}$ floor, yellow area

$12: 00-13: 00$

Poster Exhibition with Brunch

$2^{\text {nd }}$ floor, yellow area

$13: 00-15: 00$

Tutorials

$15: 00-15: 15$

Coffee Break

$15: 15-17: 15$

Tutorials

$17: 15-17: 30$

Coffee Break

$17: 30-19: 30$

Tutorials

$19: 30$

Workshop Party with Best Poster Award

Mensa Market Place, $1^{\text {st }}$ floor, yellow area 


\section{List of Overview Lectures}

\section{Monday \\ 14:00 - 16:30}

\begin{tabular}{|c|c|c|c|}
\hline $14: 00$ & $\begin{array}{l}\text { Felix Breitenecker, } \\
\text { Shabnam Tauböck }\end{array}$ & $\begin{array}{l}\text { Vienna University of } \\
\text { Technology }\end{array}$ & Welcome \\
\hline $14: 15$ & Horst Ecker & $\begin{array}{l}\text { Vienna University of } \\
\text { Technology }\end{array}$ & $\begin{array}{l}\text { Numerical investigation of the non-linear } \\
\text { rotor contacting a stator }\end{array}$ \\
\hline $14: 45$ & Karl Hollaus & $\begin{array}{l}\text { Vienna University of } \\
\text { Technology }\end{array}$ & $\begin{array}{l}\text { FEM: Domain Decomposition and } \\
\text { Homogenization for Maxwell 's Equations } \\
\text { of Large Scale Problems }\end{array}$ \\
\hline $15: 15$ & Nikolas Popper & $\begin{array}{l}\text { dwh simulation } \\
\text { services }\end{array}$ & $\begin{array}{l}\text { From Data and Structures to Models and } \\
\text { Scenarios - Future Frameworks in Decision } \\
\text { Making in Health Care }\end{array}$ \\
\hline $15: 45$ & $\begin{array}{l}\text { Markus Stöhr, } \\
\text { Stefan } \\
\text { Baumgartner }\end{array}$ & $\begin{array}{l}\text { Vienna University of } \\
\text { Technology / } \\
\text { University of Vienna }\end{array}$ & $\begin{array}{l}\text { Vienna Scientific Cluster Project - Status } \\
\text { and Applications }\end{array}$ \\
\hline \multicolumn{4}{|c|}{$\begin{array}{c}\text { Monday } \\
\text { 17:00 - 19:30 }\end{array}$} \\
\hline $17: 00$ & Thorsten Pawletta & $\begin{array}{l}\text { Wismar University of } \\
\text { Applied Sciences }\end{array}$ & $\begin{array}{l}\text { Simulation Based Evaluation and } \\
\text { Optimization of System Structures Using a } \\
\text { Graph Based Specification }\end{array}$ \\
\hline $17: 30$ & Michael Striebel & $\begin{array}{l}\text { Bergische Universität } \\
\text { Wuppertal }\end{array}$ & $\begin{array}{l}\text { Multirate and Cosimulation -- An overview } \\
\text { of current approaches and trends }\end{array}$ \\
\hline $18: 00$ & $\begin{array}{l}\text { Katharina } \\
\text { Breitenecker }\end{array}$ & $\begin{array}{l}\text { International Atomic } \\
\text { Energy Agency }\end{array}$ & On the Nature of Measurement \\
\hline $18: 30$ & Lothar Nannen & $\begin{array}{l}\text { Vienna University of } \\
\text { Technology }\end{array}$ & $\begin{array}{l}\text { Finite element methods for scattering } \\
\text { problems }\end{array}$ \\
\hline 19:00 & Dieter Suess & $\begin{array}{l}\text { Vienna University of } \\
\text { Technology }\end{array}$ & $\begin{array}{l}\text { Micromagnetic modeling of advanced } \\
\text { magnet recording structures }\end{array}$ \\
\hline
\end{tabular}

\section{Tuesday 9:00 - 11:00}

\begin{tabular}{|c|c|c|c|}
\hline 09:00 & Gertrud Konrad & $\begin{array}{l}\text { Vienna University of } \\
\text { Technology }\end{array}$ & $\begin{array}{l}\text { Modelling and Simulation of Experiments } \\
\text { and Instrumentation in Neutron Particle } \\
\text { Physics }\end{array}$ \\
\hline $09: 30$ & Giuseppe Pontrelli & $\begin{array}{l}\text { IAC-CNR, Roma } \\
\text { (Italy) }\end{array}$ & $\begin{array}{l}\text { The lattice Boltzmann method: theory, } \\
\text { computational aspects and applications }\end{array}$ \\
\hline $10: 00$ & Dirk Praetorius & $\begin{array}{l}\text { Vienna University of } \\
\text { Technology }\end{array}$ & What is Quasi-Optimality of Adaptive FEM? \\
\hline $10: 30$ & $\begin{array}{l}\text { Wolfgang } \\
\text { Kemmetmüller }\end{array}$ & $\begin{array}{l}\text { Vienna University of } \\
\text { Technology }\end{array}$ & $\begin{array}{l}\text { Mathematical Modeling and Analysis of a } \\
\text { DRT-VLF High Voltage Test Generator }\end{array}$ \\
\hline
\end{tabular}




\section{Numerical Investigation of the non-linear Rotor contacting a Stator}

Horst Ecker, Vienna University of Technology

A mathematical model to investigate the dynamics of a Jeffcott-rotor having intermittent contact with a stator is presented. The advantage of this model is found in the consistent submodel of the stator which is implemented as an additional vibratory system, interacting with the rotor model via nonlinear contact forces. Such forces are generated by a contact model, consisting of contact stiffness, damping and friction.

The contact model is activated during the contact phase which is defined by both geometrical and force-dependent conditions.

As the rotor disk and the stator are connected by a high contact stiffness during the contact phase, an integration algorithm suitable for numerically stiff systems has to be employed.

Begin and end of rotor-stator contact are defined as state events and localized by an iterative procedure.

The model allows to study the effect of the visco-elastically suspended stator on the rotor motion when intermittent contact occurs. Numerical studies are carried out for parameter variations of the rotor speed and the mass ratio of the stator and the rotor. Results are presented as Poincare'-maps and bifurcation diagrams, exhibiting rich dynamical behavior.

The numerical studies show and confirm that the damping parameter of the stator suspension has significant influence on the type of motion obtained for the rotor-stator system. As damping is decreased, periodic solutions with few contacts become less likely and nonperiodic behavior of the system dominates. 


\title{
FEM: Domain Decomposition and Homogenization for Maxwell's Equations of Large Scale Problems
}

\author{
Karl Hollaus \\ Institute for Analysis and Scientific Computing,Vienna University of Technology \\ 1040 Wien, Wiedner Hauptstraße 8-10, Austria \\ karl.hollaus@tuwien.ac.at
}

For the sake of simplicity, domain decomposition and homogenization are introduced by means of the Laplace equation, first. The basic boundary value problem of the time harmonic electromagnetic field is posed by the magnetic vector potential $A$ approximated by hierarchical vector finite elements (FEs) in $H$ (curl).

Domain decomposition methods represent a powerful tool to extend the applicability of the finite element method (FEM) to large problems, problems with moving parts, etc. and enable the use of parallel computing architectures. A novel symmetric variation formulation for the domain decomposition is presented, which belongs to hybrid mixed FEM as well as to mortaring techniques and permits the simulation of problems with non-overlapping and non-matching grids in two and three dimensions. A Helmholtz decomposition of the total vector is carried out at the interface. The scalar potential of the gradient field is represented by hierarchical FEs in $H^{1}$ introducing an additional surface space. This allows to ensure selectively the required continuity of the electromagnetic field across the interface. Spline functions of arbitrary order with $C^{n}$ continuity are used to mortar the vector and the scalar potential in the interface space. The interface is subdivided into elements similar to a FE mesh. Several numerical examples which can be easily reproduced demonstrate the versatility of the proposed method.

Multi-scale homogenization with FEM is a very promising approach to reduce the computational costs in the simulation of problems with repetitive structures, i.e. laminated materials in electrical machines, photonic crystals, metamaterials, etc. First, homogenization is demonstrated by a multi-scale approach in the context of an electrostatic problem. Then, it is extended to the eddy current problem. A multi-scale approach for $A$ has been derived to simulate the eddy current losses of a laminated iron stack. A rather coarse FE grid suffices to approximate the homogenized solution accurately. The approach consists of a $H$ (curl), a component wise $L^{2}$ and an $H^{1}$ field. The quantities in $L^{2}$ are eliminated on the FE level building the Schur-compliment efficiently. Thus, the number of unknowns of the whole system is reduced significantly. Representative numerical examples are presented to demonstrate the validity of multi-scale homogenization, the accuracy of the homogenized solution and to evaluate the computational costs. 


\title{
From Data AND STRUCTURES TO MODELS AND SCENARIOS - FUtURE FRAMEWORKS IN DECISION MAKING IN HEALTH CARE
}

\author{
N.Popper \\ dwh Simulation Services, Austria \\ 1070 Wien, Neustiftgasse 57 - 59, Austria \\ niki.popper@drahtwarenhandlung.at
}

The extraordinary high amount of 20-25 billion Euros of annual turnover in the Austrian health system has increasingly raised the questions of cost-effectiveness and evidence based decision making in health care. Health technology assessment is already intensively dealing with these issues, especially on the level of single interventions. Assessments today already often includes modeling and simulation in order to analyse long term effects, which cannot be seen directly from data or structures. This, in turn, requires increasing cooperation of experts from different fields of science such as HTA, statistics, data management, modeling \& simulation as well as visualisation. A common understanding on contents, methodology and terminology needs to be developed. In Austria, such a common understanding has hardly been elaborated in a systematic way so far.

It is the main aim of our group to support evidence based decision making in health care by service tools which helps profiting from existing potentials in HTA, modeling, simulation, statistics and data analysis. Therefore common standards will be defined in order to facilitate cooperation and interdisciplinary work. This work is done in various projects with the Main Association of Social Security Institutions or in research projects, like Innovative Framework for Evidence based Decision support in Health care (IFEDH) founded by The Austrian Research Promotion Agency (FFG). Main goal of all approaches is to define future frameworks for decision making in health care.

To achieve this goal in the future new processes for the decision support has to be developed. Especially for infectious diseases this seems to be highly effective, as results so far show. The modeling process and the generation and design of adequate modeling methods are very important. Besides this, the model and parameter validation as well as verification of the developed and implemented system are in focus. The data quality assessments as well as the outcome interpretation are set up interdisciplinary, ensuring the necessary project quality and acceptance on the part of policymakers.

Finally all these efforts have to be coordinated international. Various approaches are on their way to keep results of models and scenarios comparable, which is most important in the area of decision making in health care. One example is the coordination of all approaches with the Joint Modeling Good Research Practices Task Force of ISPOR (International Society for Pharmacoeconomics and Outcomes Research) in collaboration with The Society for Medical Decision Making (SMDM). Their goal is to ensure that good research practices on modeling techniques remain useful for all current modeling techniques as well as to foster the use of model-based results to inform health care decisions. 


\section{Vienna Scientific Cluster Project}

Dr. Markus Stöhr (HPC-Group, ZID TU Wien)

The Vienna Scientific Cluster is a collaboration of the University of Technology Vienna, the University of Vienna and the University of Natural Resources and Life Sciences Vienna (http://vsc.ac.at/).

Currently two systems are installed: VSC-1 and VSC-2. In the talk an overview of the used hard-and software will be given. 


\title{
Simulation Based EVAluation AND OPTIMIZATION OF SySTEM STRUCTURES USING A GRAPH BASED SPECIFICATION
}

\author{
T. Pawletta, O. Hagendorf \\ Wismar University of Applied Sciences, Germany

\begin{abstract}
Corresponding author: T. Pawletta, Wismar University of Applied Sciences, Research Group Computational Engineering and Automation (CEA)

23952 Wismar, Philipp-Müller-Straße, PF 1210, Germany, thor sten. pawletta@hs-wismar .de
\end{abstract}

\begin{abstract}
Complex systems are characterized by a modular, hierarchical composition of different systems and various system parameters. A frequent task in complex systems design or analysis is to determine optimal system structures and system parameters. Often modelling and simulation techniques are used to solve this task. Their usage is as diverse as the concerns of application areas. Many modelling approaches are based on the system theoretic concept of system composition and decomposition and distinguish between two fundamental system types, called atomic systems and coupled systems in [1]. Both types define input and output quantities by which they can be coupled to compose more complex systems. In addition atomic systems define a dynamic behaviour using system parameters, system states and different system functions. In contrast coupled systems define a system structure consisting of systems (sub-systems) and their coupling relations. Hence, a model is a modular, hierarchical representation of a system design that can be directly or indirectly executed by a simulator. This generic concept is independent of specific system dynamics, simulation algorithms and application areas.
\end{abstract}

A single simulation run allows the evaluation of only one system design, consisting of a specific system structure and parameter set. An established method is the integration of a simulation model with free configurable parameters in an optimization loop. Such approach allows to determine optimal parameter values of a specific system structure with regard to a defined objective function. An advanced problem is the automated evaluation of an optimal system structure including its parameter values. Prerequisites for such a holistic system design evaluation are: (i) a formal specification of all possible system designs, i.e. system structures, parameter sets, codomains, constraints etc., (ii) the definition of performance criteria and (iii) methods for an automated model generation, execution and evaluation. The System Entity Structure and Model Base (SES/MB) framework according to $[1,2]$ provides a basis for such research.

The SES is a graph based approach to specify modular, hierarchical system designs. Systems are represented using entities which may be composed, decomposed or specialized. The entities, their structural taxonomy as well as their specific properties and system design constraints are represented as a tree-graph using different types of nodes, node attributes and edges. Leaf nodes in a SES represent basic systems. These may be real atomic systems or coupled systems without any design alternatives. The MB is a library of executable models referenced by SES leaf nodes. That means the structural knowledge and all parameter information of alternative system designs are represented in a SES while the dynamic behaviour is coded in predefined basic models with free configurable parameters. Such separation allows the construction of executable models for different system designs using the same set of predefined basic models and supports a systematic analysis of design alternatives. In addition, the SES/MB framework defines methods for an automatic model generation and execution.

However, the SES/MB framework alone does not support the automatic evaluation of an optimal system design. Methods to fulfil this challenge are introduced in [3], where the SES/MB framework is integrated into an optimization environment. Such integration requires a transformation of graph-based information about design alternatives coded in a SES into parameter values adequate for an optimization algorithm. Moreover, a reinterpretation of numerical optimization results is needed to synthesize a new system design, consisting of a system structure with its parameter values, based on a subsequent SES analysis.

The fundamental ideas of the SES/MB framework and the necessary methods for its integration into an optimization environment will be discussed. Following, the usage of the advanced framework for combined system structure and parameter optimization will be demonstrated by means of engineering applications.

[1] Zeigler B.P., Praehofer H. and Kim T.G.: Theory of Modeling and Simulation. Academic Press, New York, USA, 2000.

[2] Zeigler B.P. and Hammonds P.E.: Modeling and Simulation-Based Data Engineerins. Elsevier Academic Press, Burlington, MA, USA, 2007.

[3] Hagendorf O. and Pawletta O.: A Framework for Simulation-Based Structure and Parameter Optimization of Discrete-Event Systems. In: Discrete-Event Modeling and Simulation, Ed.: Wainer, G.A. and Mosterman P.J., CRC Press Taylor \& Francis Group, Boca Raton, FL, USA, 2011, 199-222. 


\title{
Multirate and Cosimulation - An Overview of current Approaches and Trends
}

\author{
M. Striebel, S. Schöps \\ University of Wuppertal, Applied Mathematics/Numerical Analysis, Germany \\ Gaußstr. 20, 42119 Wuppertal, Germany
}

\author{
Corresponding Author: Michael Striebel \\ striebel@math.uni-wuppertal.de
}

Dynamical systems arising from industrial applications easily exhibit millions of unknowns/states. In transient analysis the systems have to be solved for these unknowns in time domain.

Classical numerical integration schemes advance the whole system, i.e., all unknowns at once, to the next time point. A time step, defining the next time point, is chosen adaptively such that each unknown satisfies specified accuracy requirements. Fast changing states ask for higher resolution in time domain time than unknowns behaving rather latent. Hence, the highly active states steer the progress of simulation and force latent states to be calculated on the same fine time grid.

Multirate integration methods allow to use different time step sizes in parallel for different groups of states. By this, simulation can be done more efficiently with respect to computational time and memory usage.

In real life problems the groups often arise naturally. In full system simulation of electrical circuits, e.g., the full system is mad up of different functional units with different behaviour. Frequently multiphysical phenomena, e.g., thermal-electric effects are of interest, too.

In this talk we present an overview of current approaches in designing efficient numerical integration schemes that take care of heterogeneous behaviour of the system to be simulated. We cover monolithic approaches based on uniform integration methods and cosimulation approaches applying the multirate idea where different physical phenomena and different types of dynamical systems require different integration schemes to be used, like e.g., in coupled device-circuit simulation. 


\section{On the Nature of Measurement}

\section{Katharina Breitenecker}

The challenge of implementing real data to mathematical models is often underestimated by programmers and software engineers. Usually, various methods and techniques of mathematical modeling and different model approaches are applied to the software that is used to program the corresponding model. However, when it comes to the evaluation of the model predictions, or whenever real world data is needed for parameter identification, the data itself is often considered as an external problem. Thus, the programmer / software engineer does not have any further knowledge about it and they concentrate just on modeling techniques and the respective software. Therefore, parameter identification or the evaluation of modeling predictions can often lead to wrong or unsatisfactory results.

Students in the disciplines of modeling and simulation usually get a good education in various modeling techniques, model approaches and software kits. However, in recent years it has been shown, that they have a good knowledge in all the theoretical aspects of programming and designing a mathematical model for various occasions; but when it comes to practical aspects, like the implementation of 'real data' to their model, students often fail due to several reasons.

Repeatedly, it has been shown that students are often not familiar with the meaning of good data quality, as 'good data quality' has been underestimated and disregarded for a long time by the software community.

This overview talk will give an introduction to the meaning of "good data quality" in order to define methods and techniques to improve this quality. It will show how data quality can be 'measured' in order to determine how valuable certain information is and how to improve it. Overall it aims at conveying a general sense about the challenges of getting good results, i.e. an estimation for the true value of a certain variable. The ideas of different scales (units) and their corresponding problems will be discussed as well as error propagation as general rules for evaluating and expressing uncertainty in measurement (i.e. GUM). 


\title{
Finite element methods for scattering problems
}

\author{
Lothar Nannen
}

Acoustic or electromagnetic scattering problems are typically modeled as partial differential equations posed on unbounded domains. Therefore, in addition to standard boundary conditions a so called radiation condition is needed to ensure that the solutions are physically relevant and unique.

In a numerical treatment of such problems with finite element methods the unbounded domain is usually splitted into a bounded interior domain, where standard methods can be used, and an unbounded exterior domain. For the exterior domain there are mainly two different concepts: Neglecting the exterior domain and introducing a so called transparent boundary condition at the artificial boundary of the interior domain or using special methods for the exterior domain.

An example of a special methods is the complex scaling method (often called perfectly matched layer method). The method introduces an artificial radial damping such that a radiating solution of the problem decays exponentially in the exterior domain. Hence, the exterior domain can be truncated to a bounded layer around the interior domain. 


\section{Micromagnetic Modeling of Advanced Magnet Recording Structures}

Presenter: Dieter Suess

The presentation starts with a short introduction into magnetic recording. Using this example a model is presented which recently became a standard tool for the calculation and optimization of recording structures - Micromagnetics. The basic equation of motion of micromagnetics, the Landau Lifshitz Gilbert equation is discussed. Methods to solve this equation are presented. Particular focus is put on a hybrid finite element / boundary element method. In addition a matrix compression technique is discussed (H-matrices) to reduce the computational complexity. 


\title{
MODELLING AND SimULATION OF EXPERIMENTS AND INSTRUMENTATION IN NEUTRON PARTICLE PHYSICS
}

\author{
G. Konrad \\ Vienna University of Technology, Austria \\ Corresponding author: G. Konrad, Vienna University of Technology, Institute of Atomic and Subatomic Physics \\ 1020 Wien, Stadionallee 2, Austria, gkonrad@ati.ac . at
}

Precision measurements of observables in neutron beta decay address important open questions of particle physics and cosmology, and are generally complementary to direct searches for new physics beyond the Standard Model in high-energy physics. Free neutron decay is therefore a very active field, with a number of new measurements underway worldwide.

The boundary element method (BEM), the finite element method (FEM), and Monte-Carlo-based (MC) codes are used to model and simulate neutron sources as well as experiments and instrumentation in neutron physics:

- to study the dynamic behavior of existing reactors and to design the next generation of nuclear reactors, space-time models are being developed in COMSOL. These models include kinetics [1], fluid flow, or heat transfer physics. The basic nuclear data are calculated with MCNP5, a MC neutron transport code.

- to precisely define and control the key parameters for the neutron beam, a novel type of spatial magnetic neutron spin resonator, MONOPOL [2], is being developed in CST EM Studio. First measurements and performance tests of a first prototype are very promising.

- to optimize existing and to design new neutron decay spectrometers, BEM, FEM and MC techniques are being used. For example, the BEM has been applied to design and optimize the charged particle spectrometer $a$ SPECT, while COMSOL has been used to search for and eliminate potential particle traps inside the spectrometer as well to study several different systematic effects. At present, the next generation of a neutron decay instrument, PERC, is being developed using BEM and FEM.

- to shield from ambient magnetic fields (with shielding factors of up to $10^{9}$ for the upcomig nEDM experiment) or to reduce the stray field in the neighborhood of a strong superconducting magnet (down to the cardiac pacemaker level for the upcoming PERC facility) sophisticated shields are being developed in COMSOL (CST EM Studio, and Radia). For the superconducting aSPECT magnet, COMSOL has been successfully used to reduce the exterior magnetic field by about a factor of 10 .

- to simulate the properties of the charged particle detectors (penetration depth, energy loss, and backscattering of the decay products), the MC toolkits SRIM, CASINO, ETRAN, or Geant4 are used. In addition, CASINO has been successfully applied to the backscattering of decay electrons from the inner surfaces of the $a$ SPECT spectrometer.

In this talk, a brief overview of the various applications of BEM, FEM, and MC techniques in neutron physics will be presented. I will compare the different methods, where applicable, and discuss the respective pros and cons.

[1] Chandler D., Freels J. D., Primm R. T., and Ivan Maldonado G.: COMSOL-Based Nuclear Reactor Kinetics Studies at the HFIR. In: Proc. COMSOL Conference, Boston, 2011, http://www.comsol.com/papers/10598/download/chandler_paper.pdf.

[2] Badurek G., Gösselsberger Ch., and Jericha E.: Design of a pulsed spatial neutron magnetic spin resonator. Physica B 406 (2011), 2458-2462.

[3] Konrad G. et al.: Design of an Anti-Magnetic Screen for the Neutron Decay Spectrometer aSPECT. In: Proc. European COMSOL Conference, Grenoble, 2007, Vol. 1, 241-245, http://cds.comsol.com/access/dl/papers/3276/Konrad.pdf. 


\section{The lattice Boltzmann method: theory, computational aspects and applications. \\ Speaker: Dr. Giuseppe Pontrelli, IAC-CNR, Roma (Italy)}

The lattice Boltzmann method (LBM) is a mesoscopic approach for modeling macroscopic hydrodynamics. It is based on a minimal kinetic Boltzmann equation in which representative particles evolve on a regular grid according to simple streaming and collision rules, designed in such a way as to preserve the basic symmetries of fluid mechanics. The LBM exhibits a number of appealing features as a computational fluid dynamics solver, such as the simplicity of the stream-and-collide kinetics, its amenability to parallel computing and its ease in handling complex flows, although the use of constant particle speeds naturally imposes a spatially uniform cartesian grid. This represents a severe limitation for many practical applications, particularly for complex geometries. To overcome this drawback, an interesting development is represented by a finite-volume formulation over unstructured grids. In blood flow problems, this allows a near-wall finer discretization that accounts for the wall undulation due to endothelial cells and captures the shear-rate dependent viscosity of blood. In this talk an overview of the LBM is given, together with such extensions and with a focus on the arterial flows. Some worked examples are shown to prove the effectiveness of this technique. 


\section{What is Quasi-Optimality of Adaptive FEM? \\ Dirk Praetorius \\ Institute for Analysis and Scientific Computing,Vienna University of Technology \\ 1040 Wien, Wiedner Hauptstraße 8-10, Austria \\ dirk.praetorius@tuwien.ac.at}

Usually the accuracy of numerical results suffers from singularities of the given data and/or the (unknown) exact solution. One remedy is to appropriately grade the underlying mesh towards these singularities. In many codes, this mesh adaptation is done automatically by use of certain (heuristic) refinement indicators. Empirically, most of these adaptive strategies perform very well in the sense that the slope of number of unknowns versus corresponding error shows the best possible decay. Such an observation is of practical relevance since each simulation aims for the best possible solution with respect to limited resources like computational time and/or memory requirements.

In recent years and for a certain class of elliptic differential equations, there has been a major breakthrough in the mathematical understanding of this empirical observation. In our talk, we aim to state "optimal convergence behaviour" of the numerical scheme in a mathematical sense. We discuss the progress made in the recent years and highlight some of the mathematical ingredients for such an optimality proof. 


\title{
Mathematical Modeling and Analysis of a DRT-VLF High Voltage Test Generator
}

\author{
W. Kemmetmüller, A. Kugi \\ Automation and Control Institute \\ Vienna University of Technology \\ \{kemmetmueller,kugi\}@acin.tuwien.ac.at
}

Due to the accelerated expansion of regenerative decentralized power generation systems, more and more high and ultra high voltage cables are used to connect the power generation systems to the power distribution system. Thus, the demand on an efficient possibility for on-site testing of cables has increased in recent years. The classical method for testing high voltage earth and sea cables is the application of a dc-voltage. This method is, however, of limited significance for evaluating the quality of a cable. Moreover, it might even damage modern cables with plastic isolation (e.g. cross-linked PEcables). Therefore, high voltage cables are preferably tested by ac-voltages. Using a frequency of $50 \mathrm{~Hz}$ or above leads, in combination with the high capacity of the cables, to an enormous reactive power which has to be provided by the power supply of the test generator. Therefore, the associated test equipment becomes very large and heavy such that on-site tests of cables become very complicated if not impossible.

For this reason, test generators with very low frequencies (VLF) are often proposed for on-site cable tests. The reduced frequency in the range of $0.1 \mathrm{~Hz}$ drastically reduces the reactive power and thus the size of the equipment, making these test methods more applicable for on-site tests of cables. Using high voltage transformers as in classical ac-test systems is, however, inefficient due to the very low frequency. This presentation deals with a novel VLF test generator, where the high voltage is generated by a series resonance circuit which is excited in its resonance frequency in the range of $1 \mathrm{kHz}$. The desired very low frequency high voltage is then obtained by a modulation of the high frequency voltage's amplitude with the desired very low frequency (DRT-difference resonance technique). This new system allows for the generation of very high voltages up to $300 \mathrm{kV}$ at a frequency of $0.1 \mathrm{~Hz}$, which is used to test long cables with a small and transportable test generator.

In this presentation, the mathematical model and the analysis of the dynamic characteristics is given. First, a detailed mathematical model of the DRT-VLF test generator is derived and its main characteristics are discussed. It is shown that this reference model allows an exact description of the system behavior. Due to its complexity it is, however, not suitable for the analysis and the controller design. Thus, an envelope model is calculated which better fits these tasks. The quality of both models is validated by means of measurement results. The second part of this presentation is concerned with the analysis of the main dynamical features of the test generator. Finally, first results of an optimal control strategy for the DRT-VLF test generator are presented. It will be shown that a significant improvement of the system's behavior can be obtained by this optimal control strategy. 


\section{List of Poster Contributions}

\begin{tabular}{|c|c|c|}
\hline Albertini Daniel & $\begin{array}{l}\text { AIT Austrian Institute } \\
\text { of Technology GmbH }\end{array}$ & $\begin{array}{l}\text { iOS Supported Real-Time Analysis of } \\
\text { Vigilance Stages Based on EOG Biosignal } \\
\text { Pattern Recognition }\end{array}$ \\
\hline Amini Ahmad & $\begin{array}{l}\text { Vienna University of } \\
\text { Technology }\end{array}$ & $\begin{array}{l}\text { Studying the Factors which Affect the Efficiency of } \\
\text { the Spinal Electromagnetic Stimulation Technique }\end{array}$ \\
\hline Anderl Daniela & University of Erlangen & Numerical Simulation of Protein Foams \\
\hline Bachler Martin & $\begin{array}{l}\text { Vienna University of } \\
\text { Technology }\end{array}$ & $\begin{array}{l}\text { Automatic detection of the QRS complex, } \mathrm{P} \text { and } \mathrm{T} \\
\text { wave in electrocardiography in real-time }\end{array}$ \\
\hline Bruckner Martin & $\begin{array}{l}\text { Vienna University of } \\
\text { Technology }\end{array}$ & Orientation Inside of a Pedestrian Flow Simulation \\
\hline Descovich Xenia & $\begin{array}{l}\text { AIT Austrian Institute } \\
\text { of Technology GmbH }\end{array}$ & $\begin{array}{l}\text { Modelling elastic walls in lattice Boltzmann } \\
\text { simulations of arterial blood flow }\end{array}$ \\
\hline $\begin{array}{l}\text { Eckhardt } \\
\text { Wolfgang }\end{array}$ & TU München & $\begin{array}{l}\text { Memory-efficient Implementation of a rigid-body } \\
\text { multi-center Molecular Dynamics Simulation for } \\
\text { Nano-Fluidics }\end{array}$ \\
\hline Englberger Julian & $\begin{array}{l}\text { UAS Regensburg / } \\
\text { IHI Zittau }\end{array}$ & $\begin{array}{l}\text { A simulation analysis of Independent Requirements } \\
\text { Consumption in Master Production Scheduling }\end{array}$ \\
\hline Erhart Jacqueline & $\begin{array}{l}\text { Vienna University of } \\
\text { Technology }\end{array}$ & $\begin{array}{l}\text { COMSOL-based Design Studies for the Upcoming } \\
\text { Neutron Decay Experiment PERC }\end{array}$ \\
\hline Führer Thomas & $\begin{array}{l}\text { Vienna University of } \\
\text { Technology }\end{array}$ & $\begin{array}{l}\text { Convergence of adaptive FEM-BEM coupling driven } \\
\text { by residual-based error estimators }\end{array}$ \\
\hline Gasser Matthias & $\begin{array}{l}\text { AIT Austrian Institute } \\
\text { of Technology GmbH }\end{array}$ & $\begin{array}{l}\text { iOS Supported Visualisation and Control of a } \\
\text { Smart-Home in the Context of AAL - Technologies }\end{array}$ \\
\hline $\begin{array}{l}\text { Godenschwager } \\
\text { Christian }\end{array}$ & $\begin{array}{l}\text { Friedrich-Alexander- } \\
\text { Universität Erlangen- } \\
\text { Nürnberg }\end{array}$ & $\begin{array}{l}\text { Simulation of Pressure Driven Blood Flow in } \\
\text { Vessels Defined by Centerlines and Radius Profiles }\end{array}$ \\
\hline Goldenits Petra & $\begin{array}{l}\text { Vienna University of } \\
\text { Technology }\end{array}$ & $\begin{array}{l}\text { Convergent Geometric Integrator for the Landau- } \\
\text { Lifshitz-Gilbert Equation with Magnetostriction }\end{array}$ \\
\hline $\begin{array}{l}\text { Hametner } \\
\text { Bernhard }\end{array}$ & $\begin{array}{l}\text { AIT Austrian Institute } \\
\text { of Technology GmbH }\end{array}$ & $\begin{array}{l}\text { Vascular Impedance Calculation Using a New } \\
\text { Blood Flow Model }\end{array}$ \\
\hline Heinzl Bernhard & $\begin{array}{l}\text { Vienna University of } \\
\text { Technology }\end{array}$ & $\begin{array}{l}\text { High-Level Modelling and Simulation of Machine } \\
\text { Tools in Energy Optimization of Production Plants }\end{array}$ \\
\hline Kovacevic Tijana & $\begin{array}{l}\text { Technische } \\
\text { Universität München }\end{array}$ & $\begin{array}{l}\text { GPU-Accelerated MRI-Simulation and Visualization } \\
\text { on a CAVE-Like Environment }\end{array}$ \\
\hline Romstorfer Georg & $\begin{array}{l}\text { Vienna University of } \\
\text { Technology }\end{array}$ & $\begin{array}{l}\text { Using Open Source Geo-Data in Agent-Based } \\
\text { Models of Health Care Utilization }\end{array}$ \\
\hline Rößler Matthias & $\begin{array}{l}\text { Vienna University of } \\
\text { Technology }\end{array}$ & $\begin{array}{l}\text { Thermodynamical Coupling of a Machine Tool with } \\
\text { its Environment }\end{array}$ \\
\hline
\end{tabular}




\title{
iOS Supported Real-Time Analysis of Vigilance Stages Based ON EOG BiOSIGNAL PATTERN RECOGNITION
}

\author{
Daniel Albertini \\ AIT - Austrian Institute of Technology, Austria \\ Corresponding author: Daniel Albertini, AIT Austrian Institute of Technology GmbH, \\ 1220 Wien, Donau-City-Straße 1, Austria, e0402305@student.tuwien.ac. at
}

Introduction Smartphones are very popular these days. They are very powerful devices and always nearby. Using this sort of devices for monitoring biosignals will become a great market in the near future. My work is to test the usage of a mobile device (iOS based) for recording and analyzing biosignals. Therefore I used the application of EOG (Electrooculography) to analyze vigilance stages.

Vigilance is the the ability to maintain attention and alertness over prolonged periods of time.

Objective Methods to Measure Vigilance.

- EEG

The alpha, beta and theta waves can be used to determine a vigilance stage.

- Mackworth Clock Test

This is a long therm reaction test where a very bored situation is simulated, which is interrupted by a random event. In most cases it is implemented as a clock where every minute a random second is skiped. The test subject has to notice this and press on a button.

- Other Biosignals

eg. Heart Rate, Eye blinking, EOG, pupillography, usw

The EOG is a technique to measure the resting potential of the retina. The eye acts as a dipol where the cornea is in positive charge and the retina has a negative charge. So with 4 electrodes around one eye you measure 2 potentials, the vertical and the horizontal potential difference.

Method The sampling frequency for the EOG signal is $200 \mathrm{~Hz}$. For the algorithms I used a 60 seconds time window. So I get 12000 values per channel. This values are used to compute the following significant vigilance parameters.

- Time Domain

Count the number of significant eye movements.

- Velocity Domain

Count significant saccade velocity overshoots. The eye

- Frequency Domain

Calculate the spectral power for every window. 


\title{
STUDYING THE FACTORS WHICH AFFECT THE EFFICIENCY OF THE SPINAL EleCtromagnetic Stimulation TeChNiQue
}

\author{
A. Amini, K. Minassian ,F. Rattay \\ Vienna University of Technology, Austria \\ Corresponding author: A. Amini, Vienna University of Technology, Institute for Analysis and Scientific \\ Computing \\ 1040 Wien, Wiedner Hauptstraße 8 - 10, Austria, ahmad.amini@tuwien.ac.at
}

\begin{abstract}
Introduction. The main aim of this study is to provide a stimulation technique which helps the spinal cord injured (SCI) persons, by means of reanimating the paralyzed lower spinal cord, since injuries to the spinal cord interfere with electrical signals between the brain and the muscles, resulting in paralysis below the level of injury. Functional electrical stimulation (FES) or neuromuscular electrical stimulation (NMES) is a technique that uses electrical currents to activate nerves innervating extremities affected by paralysis resulting from spinal cord injury (e.g., with implanted and surface electrodes [1]). Restoration of limb function as well as regulation of organ function are the main application of FES, although FES is also used for treatment of pain, pressure, sore prevention, etc. However, in this study we only focus on applications that may help these patients via controlling the everyday stepping and standing-like movements.
\end{abstract}

But FES-users with high-sensitivity may feel some discomfort during treatment, such as "pins and needles" under their skin, and a tingling sensation caused by the flow of electrical currents passing through their body. A majority of users will overcome these sensations, but it is still extremely important that the device is tuned to the user's comfort level (i.e., current type, modulation, waveform, pulse duration and repetition rate, and intensity) or treatment may be unsuccessful. On rare occasions the FES electrode's adhesive or gel may cause users to develop skin irritation and rashes. Most often, this is addressed by replacing the electrodes with a hypoallergenic version. Individuals with cardiac pacemakers or implanted defibrillators should be extremely cautious using FES-type devices and should consult their doctors before beginning treatment. FES treatment should not be administered to women who are pregnant; or individuals with congestive heart failure (blood clots or circulation issues); cardiac arrhythmias; chronic or severe kidney or lung diseases; active and non-healing wounds; severe muscle contractions; tendency for worsening AD due to FES; or sensitivity to electrodes. ([2] And also references mentioned there)

On the other hand, the spinal electromagnetic stimulation (SEMS) is a non-invasive painless stimulation method in which a magnetic field is generated by a magnetic coil in an interested area of the spinal cord. This magnetic field induces an electric field [3] which modulates neuronal activity. Magnetic stimulation of the spinal cord produces less pain at the same level of isometric peak torque than did FES. Magnetic stimulation is a potential alternative to FES, especially for persons with intact or residual sensory function.

The spatial distribution of the induced electric field is determined by two important factors. First: the coil properties (i.e., geometry, position, current waveform and etc.), second: the body tissues dielectric properties (i.e., conductivity and permittivity) [4]. To study these factors, a simplified model of the body with three different tissues (i.e., general thorax, vertebra and spinal cord) and two different coils (i.e., 8-shaped and round coils) is simulated. In order to calculate the induced electric field through each tissue, two analysis software (i.e., COMSOL and SEMCAD) are simultaneously used. The final simulated results identify, which one of these coils and also which stimulation points of the body, are the most effective choices.

Index Terms. Spinal cord injury (SCI), Magnetic stimulation, 8-shaped coil, Round coil, Electrical conductivity, Vertebra.

[1] Ladenbauer J., Minassian K., Hofstoetter U.S., Dimitrijevic M.R. and Rattay F.: Stimulation of the Human Lumbar Spinal Cord With Implanted and Surface Electrodes: A Computer Simulation Study. IEEE Transactions on Neural Systems and Rehabilitation Engineering, 18(6), (2010), 637-645.

[2] Han TR., Shin HI. and Kim IS.: Magnetic stimulation of the quadriceps femoris muscle: comparison of pain with electrical stimulation. Am J Phys Med Rehabil., 85(7), (2006), 593-9.

[3] Jackson JD.: Classical Electrodynamics,3re ed. Wiley \& Sons, New York, 1999.

[4] Efthimiadis K.G., Samaras T. and Polytzoidis K.S.: Magnetic stimulation of the spine: the role of tissues and their modelling. Phys. Med. Biol., 55, (2010), 2541-2553. 


\title{
NUMERICAL SimULATION OF PROTEIN FOAMS
}

\author{
D. Anderl, S. Donath, C. Rauh, U. Rüde, A. Delgado \\ Friedrich-Alexander-Universität Erlangen-Nürnberg, Germany
}
Corresponding author: D. Anderl, Friedrich-Alexander-Universität Erlangen-Nürnberg, Institute for Fluid Mechanics
91058 Erlangen, Cauerstraße 4, Germany, daniela.anderl@lstm.uni-erlangen.de

In industrial applications desired and undesired foam production in fluids containing proteins is an issue. To gain basic understanding of this phenomenon simulation tools are demanded. Proteins affect the stability of the foam lamellas and influence the rheology of a gas dispersion in general. Here the proteins under considerations are $\beta$-Casein and $\beta$-Lactoglobulin. In this contribution the modeling of foam behavior under the influence of proteins is attacked by simulations. In order to validate the simulation results, experiments are carried out.

The principle to model foaming is based on the free surface method by Körner et al. [1]. This is not a classical multiphase method but only the liquid phase is simulated and special boundary conditions account for the gas phase. This method is integrated in the Lattice Boltzmann framework waLBera (widely applicable Lattice Boltzmann solver from Erlangen, own development of Institute for System Simulation at Friedrich-Alexander-Universität Erlangen-Nürnberg). This software framework can be used massively parallel which allows to simulate large bubble assemblies. One possibility to account for the surface interaction due to protein adsorption is to introduce a disjoining pressure. This additional pressure, which adds to the bulk pressure, comes into play whenever small gaps appear and the corresponding boundaries interact. It accounts for the forces which arise due to interactions between the proteins at the surfaces. To model this correctly, information about the distances between the bubbles is needed in the simulation. The method which calculates the bubble distances works in a diffusive manner and allows therefore parallel computations [2]. With this method the effect of this extra surface force is evaluated.

One application in this contribution is the correct treatment of a bubble break-up which allows to simulate the foaming process by a membrane. Here it is necessary that bubbles can detach from the pores in a membrane. Those bubbles have to be initialized with a correct pressure which is an algorithmical challenge here. The detachment process is validated with an analytical model. The correct behavior of the bubbles rising through a membrane is necessary to compare simulation results with experiments which are done with a standard glass drip.

Furthermore the surface tension is changed according to a theoretic model [3]. Here, the focus is on a realistic modeling of the surface tension accuracy when proteins adsorb at the water-gas-interfaces. The adsorbed proteins decrease the surface tension and therefore stabilize the foam lamellas. The adsorption process starts when a new surface is created and can be modeled as irreversible and diffusion-controlled [4].

[1] C. Körner, M. Thies, T. Hofmann, N. Thürey, and U. Rüde: Lattice Boltzmann Model for Free Surface Flow for Modeling Foaming. Journal of Statistical Physics, Vol 121, Nos. 1/2, October 2005.

[2] T. Pohl: High Performance Simulation of Free Surface Flows Using the Lattice Boltzmann Method. Dissertation

[3] V.B. Fainerman, E.H. Lucassen-Reynders, R. Miller: Description of the adsorption behavior of proteins at water/fluid interfaces in the framework of a two-dimensional solution model. Advances in Colloid and Interface Science, 106 (2003) 237-259.

[4] J. Benjamins, A. De Feijter, M.T.A. Evans, D.E. Graham, and M.C. Phillips: Dynamic and Static Properties of Proteins Adsorbed at the Air/Water Interface. Faraday Discuss. Chem. Soc., 1975, 59, 218-229. 


\title{
AUTOMATIC DETECTION OF THE QRS COMPLEX, P AND T WAVE IN ELECTROCARDIOGRAPHY IN REAL-TIME
}

\author{
M. Bachler, C. Mayer, B. Hametner, S. Wassertheurer \\ AIT Austrian Institute of Technology GmbH
Corresponding author: M. Bachler, AIT Austrian Institute of Technology GmbH, Health \& Environment Department \\ 1190 Wien, Muthgasse 11, Austria, martin.bachler.fl@ait.ac .at
}

\begin{abstract}
Motivation. Cardiovascular diseases are amongst the most common diseases and the leading cause of death in developed countries. The earlier these diseases are diagnosed, the better is the success rate of the treatment and the prognosis. Dynamic changes in the duration of certain intervals (shown in the figure to the right) in the electrocardiograph (ECG) are well established indicators in the diagnosis of cardiac diseases. Automating the measurement process of the ECG and combining it with its analysis yields numerous advantages over manual methods, therefore an embedded system for data acquisition, processing and analysis was developed.
\end{abstract}

Development process. The first phase of the development process consisted of the creation of an algorithm using MATLAB ${ }^{\circledR}$ and its verification against ECG signals manually annotated by medical experts using PhysioNet databases [1]. During the second stage, the algorithm was ported to the embedded System using Embedded MATLAB ${ }^{\circledR}$, followed by a hardware-in-theloop simulation coupling the measurement hardware with the MATLAB ${ }^{\circledR}$ model in order to validate the developed system.

The algorithm. The algorithm presented in this work detects $\mathrm{R}$ peaks based on the signals amplitude and first derivative [2]. False positive detections due to artefacts are prevented by analysing the signal's local statistic characteristics. These intermediate results are classified in real-time to distinguish normal heartbeats from potential premature ventricular contractions. Templates are built from each class of heartbeats to reduce noise. These are used for the detection of the QRS complex, P and T wave. These characteristic features of the ECG are detected by the first derivatives of their class-templates [3] and a geometric method, respectively. Finally, they are separately refined for each detected heartbeat.

Results. In the verification phase as well as in the hardware-in-the-loop simulation, the algorithm achieved a sensitivity of $98.2 \%$ and a positive predictive value of $98.7 \%$, respectively. Its accuracy, shown in the figure below, matches those of human medical experts, so one can conclude that its performance is adequate for the calculation of certain relevant intervals of the ECG.

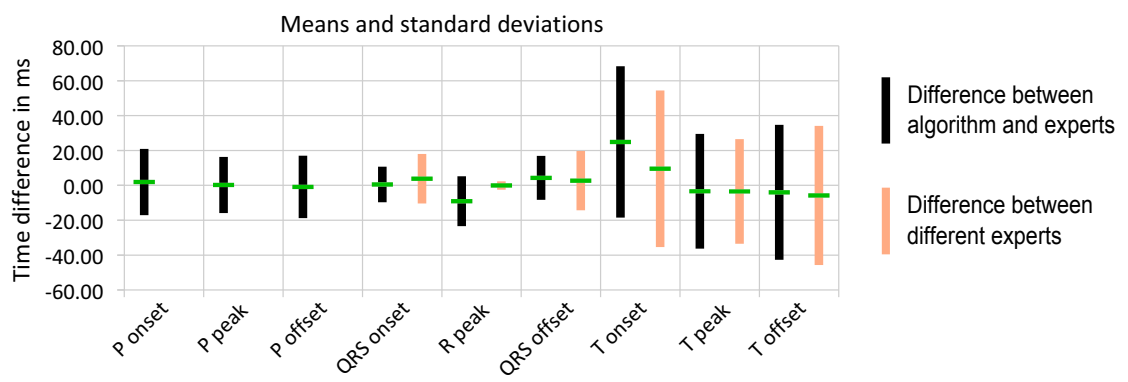

The accuracy of the presented algorithm, measured as difference between the algorithm's and the expert's results, compared to the uncertainty of measurement among medical experts, in terms of means and standard deviations.

[1] A.L. Goldberger, L.A.N. Amaral, L. Glass, J.M. Hausdorff, P.Ch. Ivanov, R.G. Mark, J.E. Mietus, G.B. Moody, C.-K. Peng, and H.E. Stanley: PhysioBank, PhysioToolkit, and PhysioNet: Components of a New Research Resource for Complex Physiologic Signals. Circulation 101 (2000), e215-e220.

[2] V.S. Chouhan, and S.S. Mehta: Detection of QRS complexes in 12-lead ECG using adaptive quantized threshold.. IJCSNS 8 (2008), 155-63.

[3] I. Christov, and I. Simova: Fully automated method for QT interval measurement in ECG. Computers in Cardiology (2006), 321-324. 


\title{
ORIENTATION INSIDE OF A PEDESTRIAN FLOW SIMULATION
}

\author{
M. Bruckner ${ }^{1}$, S. Tauböck ${ }^{1}$, N. Popper $^{2}$ \\ ${ }^{1}$ Vienna University of Technology, Austria; \\ 2"die Drahtwarenhandlung" - Simulation Services, Vienna, Austria \\ Corresponding author: Martin Bruckner ${ }^{1}$ \\ martin.bruckneratuwien.ac.at
}

\begin{abstract}
Introduction: This Abstract discusses algorithms that support virtual entities to navigate through a building which is modelled using an agent based system (AB). The main component of this model is the discrete grid that contains the basic structure of the building in its cells. Each of them can be, for example, a corridor, a wall, a staircase, and so one. Because it is very difficult to model a $3 \mathrm{D}$ building using only one discrete grid, each floor is mapped to its own grid; these grids are connected with special transformation areas at the end of the stairs. To simplify a student's life they need a plan how to get from point A to point B using the shortest way possible. Basically we distinguish between two algorithms: One for the global routing, which supplies a list of intermediate waypoints and one for the local routing, which directs the entity from one of these waypoint to the next.
\end{abstract}

Local Routing: For the local routing algorithm a static floor field is used. This is, as the name suggests, a field of static values, which contains the distances to the various waypoints. This field is embedded into the AB. So it is possible to "ask" each cell "how far is it to the door with name doorLectureRoom8". To determine now the shortest path, the associated cells will be scanned for each direction and afterwards the arithmetical average is calculated. So the direction with the smallest distance is to favor for the next step. To initialize this data structure, a modified variation of the Flood Fill algorithm is used. This is a simple procedure from Computer Graphics, to colorize a bordered area. The first step is the detection of all waypoint cells which

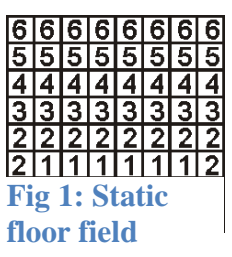
are in direct neighbourhood from a corridor, or a stair cell. So each of them is starting point for the floor fill algorithm and initialises with the distance zero. Afterwards the eight neighbourhood cells are visited and the distance value will be updated if the new value is smaller than the current value. This procedure goes one recursive so that the distance values fill the whole discrete grid.

Global Routing: For the global routing algorithm an undirected and weighted graph will be generated with help of the static floor field. Now, using this graph and the Dijkstra's algorithm a waypoint list with the shortest or fastest way will be created. Shortest and fastest way does not necessarily have to be always the same, especially special in case of using elevators. Figure 2 depictures the underlying idea of this graph. It is obviously that in on discrete grid each waypoint is connected with each other and only the change areas give the Dijkstra algorithm the possibility to jump from one grid to another.

- Node: Basically there exist only two types of nodes: doors and change-areas which include also the elevator-areas. In case of doors only the entrance of the lecture-rooms and building-exits are considered. All other doors, such as fire doors or corridor doors are generally disregarded. Even the smallest narrowing in a corridor proved to hinder people's movement significantly. The change-areas give the individuals the ability to change the discrete grids, for example at changing floors at the end of a stair or using an elevator

- Edges: In every $\mathrm{AB}$ each node is connected via edges with every other one, whereby each edge has two weights, the distance in meter and the time needed by an average human to walk this way without obstructions.

Routing: Now if a new entity is inserted into the simulation the nodes with normally represent the start and finish rooms are picked out of the graph. Afterwards a storage with all, until now, calculated ways through the building will prompted if the calculating waypoint list is already deposited. This search needs only a short time but can help to avoid an expansive search process. If the way does not exist already the Dijkstra algorithm calculates and forwards it to the entity and saves it afterwards in the before mentioned storage. In this list the first and the last waypoint are the start and finish rooms. Now it is possible for the entity to start at the grid were the start node is located and "ask" the grid for the second waypoint. If they reach it there are only two possibilities: the node can be the finish waypoint or it is a change area that transports them to another grid where they, after updating their waypoint to node three, continue moving along. So with these two algorithms (global and local) it is possible to reach each target room in a building in the shortest or the fastest way, depending on the selected edge-weight of the Dijkstra's Algorithm. 


\title{
Modeling Elastic Walls in Lattice Boltzmann Simulations of ARTERIAL BLOOD FLOW
}

\author{
X. Descovich, G. Pontrelli, S. Succi, S. Melchionna, M. Bammer \\ Vienna University of Technology, Austria \\ Consiglio Nazionale delle Ricerche, Italy \\ AIT Austrian Institute of Technology GmbH, Austria \\ Corresponding author: X. Descovich, AIT Austrian Institute of Technology GmbH \\ 1190 Wien, Muthgasse 11, Austria, xenia.descovich.fl@ait.ac.at
}

Introduction. An essential part in the simulation of blood flow in arteries is the incorporation of the arterial elasticity by modeling the vessel wall and its interaction with the fluid inside the vessel. Common numerical methods for blood flow simulations with elastic walls are complex. We suggest a simple approach for modeling elastic walls in lattice Boltzmann (LB) simulations of arterial blood flow. Our model fulfills the essential properties of an elastic wall and respects the basic conservation laws.

Modeling elastic walls in lattice Boltzmann simulations. In simulations of blood flow, it is important to consider the compliance of the vessel. Therefore, a model for the vessel wall is needed that describes its spatial displacement as it interacts with the flow dynamics. Based on [1], we have developed a model which does not need a parametrization of the wall. The method acts strictly locally, like the LB method, so that the complexity of the algorithm is low.

The model uses a lattice of nodes that can have two different states: fluid, representing the blood inside the vessel, and solid, describing the vessel wall. The compliance of the wall is modeled by changing the type of a node - from solid to fluid in the case of expansion and vice versa in the case of contraction of the vessel. This change of node type is dependent on the local pressure of the surrounding fluid nodes. Pressure thresholds are assigned to each node, increasing with the radius of the vessel segment (based on a linear relationship between the pressure and the radius).

Improved method to model elastic vessel wall. In our model, contrary to [1], the wall of the vessel is not situated on the solid nodes but is imagined to be located between last fluid and first solid node in a given direction. All nodes that are not fluid are by default solid. Thus, the problem of rupture of the vessel wall does not occur and our approach does not require the use of cellular automata. Created fluid nodes need to be initialized, which is done by averaging the LB populations from the fluid nodes surrounding the new fluid node. Compared to the method of [1], where new fluid nodes are initialized with an equilibrium distribution function, this approach includes also the non-equilibrium part of the populations, which is not negligible for nodes in proximity of the wall.

Furthermore, mass is a priori not conserved when the total number of fluid nodes increases or decreases (its circumvention is not detailed in [1].) In order to ensure mass conservation, we developed two methods that rescale the LB populations when a node type change occurs. The method of local rescaling takes into account only the nearest neighbors of the node changing its state. It redistributes mass between nodes that change state and their neighboring nodes. The method of rescaling 'by columns' takes into account the whole column of nodes - the vessel can be considered as a sequence of 'rings' adjoint to each other - in which a node type change occurs. When a node changes its state, mass is redistributed along all nodes in the same column.

Simulation and preliminary results. We implemented a simulation software for the lattice Boltzmann algorithm in two dimensions combined with our elastic wall model using the programming language $\mathrm{C}$. The program includes the rescaling methods and the pressure threshold algorithm described in the full paper. Using our software program, we conducted numerical experiments to show the feasibility of our approach.

First, we compared the computed velocity profile in a straight channel to the analytical solution of a Poiseuille flow showing that both velocity profiles coincide. Second, we tested our modeling of elasticity using local rescaling, showing that mass is properly conserved and initial values of the density and the velocity are recovered after one cycle of expansion and subsequent contraction. Third, we tested the modeling of elasticity using rescaling 'by columns' which minimizes local perturbations but exhibits the drawback that node type changes at one wall boundary affect the flow field within the whole channel, which does not correspond to real fluid dynamics.

Outlook. The aim of the future work is the simulation of blood flow in stented arteries. The approach for the modeling of elastic walls presented in this work has the advantage that it can also be extended to include stents. This enhanced model will be implemented in our simulation software and further elaboration of our approach will be reported in a later work.

[1] Leitner D.: Simulation of Arterial Blood Flow with the Lattice Boltzmann Method. Ph.D. thesis, Vienna University of Technology, 2007. 


\title{
MEMORY-EFFICIENT IMPLEMENTATION OF A RIGID-BODY MULTI-CENTER MOLECUlar DYNAMICS SIMULATION FOR NANO-FLUIDICS
}

\author{
W. Eckhardt \\ Technische Universität München, Germany \\ Corresponding author: W. Eckhardt, Technische Universität München, Chair for Scientific Computing \\ 85748 Garching, Boltzmannstraße 3, Germany, eckhardw@in.tum.de
}

\begin{abstract}
Motivation. The memory footprint of molecular dynamics simulations (MD) is important on systems with accelerator hardware such as GPGPUs or on systems like BlueGene with as little as $512 \mathrm{MB}$ memory per core. This holds especially for coupled molecular dynamics lattice boltzmann simulations [1] whith high memory demands. We present an implementation with low memory footprint for rigid-body multi-centered MD in the programme package 1s1/Mardyn [2].
\end{abstract}

Rigid-body multi-center molecular dynamics simulation. We model a fluid as a system of $N$ discrete particles, where each particle has multiple interaction sites which are fixed relative to the center and orientation of the molecule. All molecules $i$ and $j$ impact each other through pairwise interaction of their sites resulting in a force $F_{i j}=\sum_{n \in \text { sites }_{i}} \sum_{m \in \text { sites }_{j}} F_{n m}\left(r_{n m}\right)$ and torque $\tau_{i j}=\sum_{n \in \text { sites }_{i}} \sum_{m \in \text { sites }_{j}} r_{n m} \times F_{n m}\left(r_{n m}\right)$, where $r_{n m}$ is the distance of the sites. A molecule type modelling a given matter may have any arbitrary number of centers of different potential types, e.g. Lennard-Jones or dipoles. Typically, for short-range potentials like the Lennard-Jones potential a cutoff radius $r_{c}$ is introduced and the contributions of distant particles is neglected. This can be efficiently implemented with the Linked-Cell algorithm, where the computational domain is subdivided in cells of length $r_{c}$. In order to find the neighbours of a given molecule, only the cell of the molecule and its 26 adjacent cells have to be searched, reducing the overall complexity of the force calculation from $O\left(n^{2}\right)$ to $O(n)$. As most memory is dedicated to the storage of particle data, we developed and evaluated two implementation alternatives and combined them to gain memory efficiency while maintaining good performance.

\begin{tabular}{l|c|c|c|c} 
& & $\begin{array}{c}\text { Argon } \\
\text { (1CLJ) }\end{array}$ & $\begin{array}{c}\text { Ethylenoxide } \\
\text { (3CLJD) }\end{array}$ & $\begin{array}{c}\text { Benzene } \\
\text { (6CLJ6Q) }\end{array}$ \\
\hline \multirow{2}{*}{ Alt. A } & SP & 192 & 276 & 528 \\
& DP & 292 & 460 & 964 \\
\hline \multirow{2}{*}{ Alt. B } & SP & 88 & 88 & 88 \\
& DP & 164 & 164 & 164
\end{tabular}

Table 1: Memory requirement in Bytes per molecule for different test fluids.

\begin{abstract}
Alternative A. Similar to the fly-weight design pattern, each molecule can be described by position, velocity, orientation, angular velocity, an id, and a flag indicating the type of the molecule, i.e. its interaction centers. Additionally, force and torque have to be stored, resulting in a memory consumption of 88 Bytes (single precision) or 164 Bytes (double precision) per molecule. During the interaction calculation the force and torque are accumulated for the molecule instantaniously. However, for each site-site interaction, the positions of the sites have to be calculated from the center and orientation of the molecule, causing a considerably larger runtime.

These multiple redundant calculations are avoided by alternative B. For each molecule the positions of its interaction sites as well as for oriented sites like dipoles the orientations are calculated one and stored explicitely at the beginning of an iteration. During the calculation of the interactions, the force is being accumulated for each site seperately, and only at the end of the force calculation the total force effective on the molecule as well as the torque is calculated. As the number of interaction sites per molecule is not known at compile time, the addidional temporary memory has to be allocated dynamically, so each molecule consumes memory for pointers as well as the memory allocated if needed, but the runtime benefits.
\end{abstract}

Hybrid Scheme. We combined both approaches to form a memory efficient implementation at even slightly better performance. Initially, we store all molecules as described as alternative A. When a cell is first searched for interacting particles, the molecules of that cell are converted into representation B (marked as AB). Similarily, when a cell is searched for the last time, its molecules are converted back to the memory efficient representation A (marked as BA). This preserves the good performance of scheme B at low memory footprint, which is now quasi-independent of the material being simulated. Moreover any kind of data reordering fits naturally into the pattern suggested opening the doorway to efficient implementations such as vectorization.

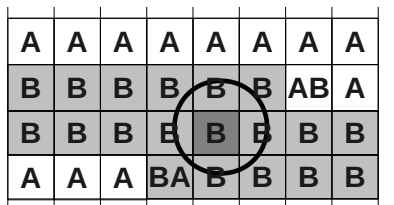

Figure 1: Molecule representation during the cell traversal.

[1] Neumann P., and Hoffman P., and Eckhardt W., and Harting J.: All Good Things Come in Threes: Current Software and Algorithmic Developments for Molecular Dynamics-Lattice Boltzmann Simulations. Poster; Cecam Workshop 2011: Multiscale Modelling of Simple and Complex Liquid Flow Using ParticleContinuum Hybrids. http: //www5. in . tum. de/pub/neumann_cecam2011.pdf.

[2] Buchholz, M. and Bungartz, H.-J. and Vrabec, J: Software design for a highly parallel molecular dynamics simulation framework in chemical engineering. In: Journal of Computational Science, 2 (2011), 124-129. 


\title{
A simulation analysis of Independent Requirements Consumption in Master Production Scheduling
}

\author{
Julian Englberger ${ }^{1}$, Prof. Dr. Frank Herrmann ${ }^{2}$, Prof. Dr. Thorsten Claus ${ }^{3}$ \\ ${ }^{1}$ Innovation Center for Production Logistics and Factory Planning (IPF), University of Applied Sciences Regens- \\ burg \\ ${ }^{2}$ Innovation Center for Production Logistics and Factory Planning (IPF), University of Applied Sciences Regens- \\ burg \\ ${ }^{3}$ Chair of Production Management and Information Technology, IHI Zittau
}

\begin{abstract}
This poster presents purpose and function of the Independent Requirements Consumption in Master Production Scheduling and gives recommendations for its application. The approach of hierarchical production planning and its planning problems are explained. The problem of new customer requirements information between planning periods and solving options are shown. The solving option of Independent Requirements Consumption is focus of this poster. The algorithm of the Independent Requirements Consumption that is implemented in commercial Enterprise Resource Planning (ERP) systems like the SAP system is analyzed. The methods for analyzing the effects of the Independent Requirements Consumption are outlined. The scenarios for simulation studies are stated and compared to similar investigations. The simulation studies were performed on a simulation system at IPF that contains both a production planning and control system and a production. The extent of simulation analyses outreaches similar investigations, especially because of the variety of scenarios. For scenarios in which capacity is available to a sufficient extent, advice for setting the Independent Requirements Consumption parameters is given. It is pointed out that with production constrained scenarios, the necessity to level out production leads to problems. An algorithm to determine necessary inventory levels is presented. It is stated that straight advice for Independent Requirements Consumption parameter settings might not be possible with production constrained scenarios. Further analyses with production constrained scenarios as well as investigations concerning safety stocks in combination with Independent Requirements Consumption are current and future research topics at the Innovation Center for Production Logistics and Factory Planning.
\end{abstract}




\title{
COMSOL-BASED Design StUdies For THE UpCOMING NeUtron DECAY EXPERIMENT PERC
}

\author{
J. Erhart, H. Abele, G. Konrad \\ Technische Universität Wien, Österreich \\ Corresponding author: J. Erhart, , Atominstitut \\ 1020 Wien, Stadionallee 2, Austria, jerhart@ati . ac . at
}

We present a new facility, PERC (Proton and Electron Radiation Channel), for the investigation of neutron beta decay. Neutron decay experiments play an important role in testing the Standard Model of particle physics and searching for new physics beyond it. These experiments are complementary to direct searches in high energy physics. PERC is designed as a source of neutron decay products, namely electrons and protons. The charged decay products are collected by a strong magnetic field directly from inside a neutron guide. The superconducting magnet system of PERC will consist of three parts on a total length of 11.5 meters. The outer parts are designed as single long solenoids with a length of $8 / 1.5$ meters and a magnetic flux density of $1.5 / 0.5$ Tesla, respectively. In contrast, the central part consists of a complex system of 8 coils in total. Short tilted coils serve to decouple the neutron beam from its decay products, whereas a solenoid part serves to limit the phase space. Here, the magnetic field varies from 3 to 6 Tesla. PERC is designed to considerably improve the precision of existing neutron decay experiments. Therefore, the magnetic field has to be known with a relative uncertainty of $10^{-4}$.

Depending on the decay parameters studied, the analysis of the decay products must be performed with different and specialized detectors. Therefore, an electron energy spectrometer, a proton retardation spectrometer and a magnetic spectrometer are still under development. Besides the electro-/magnetic field calculations, particle tracking simulations are performed in order to understand the behavior of the charged particles in those spectrometers.

In addition, in order to reduce the stray field in the neighborhood of the PERC magnet down to the cardiac pacemaker level, a magnetic field return is under development.

We have designed the magnetic field of the superconducting PERC magnet using COMSOL Multiphysics. The AC/DC module was applied to

- verify the homogeneity constraints of the magnetic field and

- reduce the exterior magnetic field down to cardiac pacemaker level.

Additionally, the Particle Tracing module was used to

- calculate the charged particle beam behavior in PERC and its different post-spectrometers.

[1] Dubbers D. et al.: A clean, bright, and versatile source of neutron decay products. Nucl. Instr. Meth. A 596, 238-247, 2008. 


\title{
Convergence of adaptive FEM-BEM coupling driven by residual-based error estimators
}

\author{
M. Aurada, M. Feischl, T. Führer, M. Karkulik, M. Melenk, D. Praetorius \\ Institute for Analysis and Scientific Computing, TU Vienna \\ Wiedner Hauptstrasse 8-10, A-1040 Vienna \\ thomas.fuehrer@tuwien.ac.at
}

Model problem \& discretization. Let $\Omega \subseteq \mathbb{R}^{2}$ be a bounded Lipschitz domain with polygonal boundary $\Gamma$ and diameter $\operatorname{diam}(\Omega)<1$. We consider a linear interface problem which can equivalently be stated via the Johnson-Nédélec FEM-BEM coupling method: Find $(u, \phi) \in \mathcal{H}:=H^{1}(\Omega) \times H^{1 / 2}(\Gamma)$ such that

$$
\begin{aligned}
\langle\nabla u, \nabla v\rangle_{\Omega}-\langle\phi, v\rangle_{\Gamma} & =\langle f, v\rangle_{\Omega}+\left\langle\phi_{0}, v\right\rangle_{\Gamma}, \\
\left\langle\psi,\left(\frac{1}{2}-K\right) u\right\rangle_{\Gamma}+\langle\psi, V \phi\rangle_{\Gamma} & =\left\langle\psi,\left(\frac{1}{2}-K\right) u_{0}\right\rangle_{\Gamma},
\end{aligned}
$$

holds for all $(v, \psi) \in \mathcal{H}$. The problem (1) admits a unique solution, if the given data $f \in L^{2}(\Omega)$, $u_{0} \in H^{1 / 2}(\Gamma)$ and $\phi_{0} \in H^{-1 / 2}(\Gamma)$ fulfill $\langle f, 1\rangle_{\Omega}+\left\langle\phi_{0}, 1\right\rangle_{\Gamma}=0$. We use a lowest-order Galerkin scheme to discretize problem (1), i.e. we replace $\mathcal{H}$ by the discrete space $\mathcal{X}_{\ell}:=\mathcal{S}^{1}\left(\mathcal{T}_{\ell}\right) \times \mathcal{P}^{0}\left(\mathcal{E}_{\ell}^{\Gamma}\right)$, where $\mathcal{S}^{1}\left(\mathcal{T}_{\ell}\right)$ denotes the space of piecewise affine and globally continuous functions and $\mathcal{P}^{0}\left(\mathcal{E}_{\ell}^{\Gamma}\right)$ is the space of piecewise constants on the boundary. Here, $\mathcal{T}_{\ell}$ is a regular triangulation of $\Omega$ and $\mathcal{E}_{\ell}^{\Gamma}$ denotes the set of all boundary edges of $\mathcal{T}_{\ell}$.

Convergence of adaptive algorithm. We use the reliable edge-based residual error estimator $\rho_{\ell}$ from [2] to steer a common adaptive mesh-refinement of the type

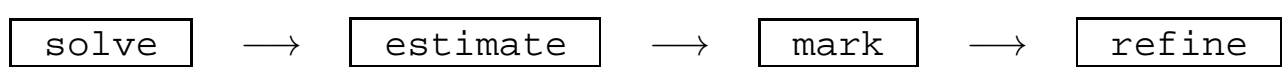

We follow the concept of estimator reduction from [3]: Suppose that Dörfler marking is used to mark edges for refinement and that at least all marked edges are refined to obtain the new mesh $\mathcal{T}_{\ell+1}$. Then, the residual error estimator satisfies the reduction estimate

$$
\rho_{\ell+1}^{2} \leq \kappa \rho_{\ell}^{2}+C\left(\left\|u_{\ell+1}-u_{\ell}\right\|_{H^{1}(\Omega)}^{2}+\left\|\phi_{\ell+1}-\phi_{\ell}\right\|_{H^{-1 / 2}(\Gamma)}^{2}\right)
$$

with certain $\ell$-independent constants $0<\kappa<1$ and $C>0$. Here, $\left(u_{\ell}, \phi_{\ell}\right) \in \mathcal{X}_{\ell}$ denotes the Galerkin solution on the mesh $\mathcal{T}_{\ell}$. Now, the estimator reduction (3) and elementary calculus prove $\rho_{\ell} \rightarrow 0$ as $\ell \rightarrow \infty$. Therefore, the reliability of $\rho_{\ell}$ shows convergence of the adaptive algorithm, i.e., convergence of $\left(u_{\ell}, \phi_{\ell}\right)$ to the exact Galerkin solution $(u, \phi)$ as $\ell \rightarrow \infty$.

Innovations. The main ingredient to prove (3) for residual error estimators are the following novel inverse-type estimates for the integral operators associated with the Laplacian, cf. [1, 4]: There is a constant $C_{\text {inv }}>0$ such that

$$
\begin{gathered}
\left\|h_{\ell}^{1 / 2}\left(V \psi_{\ell}\right)^{\prime}\right\|_{L^{2}(\Gamma)}+\left\|h_{\ell}^{1 / 2} K^{\prime} \psi_{\ell}\right\|_{L^{2}(\Gamma)} \leq C_{\mathrm{inv}}\left\|\psi_{\ell}\right\|_{H^{-1 / 2}(\Gamma)}, \\
\left\|h_{\ell}^{1 / 2}\left(K v_{\ell}\right)^{\prime}\right\|_{L^{2}(\Gamma)}+\left\|h_{\ell}^{1 / 2} W v_{\ell}\right\|_{L^{2}(\Gamma)} \leq C_{\mathrm{inv}}\left\|v_{\ell}\right\|_{H^{1 / 2}(\Gamma)},
\end{gathered}
$$

holds for all discrete functions $\psi_{\ell} \in \mathcal{P}^{0}\left(\mathcal{E}_{\ell}^{\Gamma}\right)$ and $v_{\ell} \in \mathcal{S}^{1}\left(\mathcal{T}_{\ell}\right)$. Here $h_{\ell}$ denotes the local mesh-width function.

Generalizations. The results above still hold true for other (un-)symmetric coupling methods in $2 \mathrm{D}$ or $3 \mathrm{D}$ as well as for certain types of nonlinearities in the interior domain $\Omega$. Furthermore, higher order functions can be considered as ansatz- and test-functions in the discrete Galerkin scheme. 
Applications. A broad range of physical problems can be described as transmission problems, and the variational formulation (1) can be used to obtain a numerical solution. In particular, we consider the computation of the magnetostatic potential, which plays an important role in micromagnetics and usually is a bottleneck in numerical simulations of micromagnetic phenomena.

\section{References}

[1] M. Aurada, M. Feischl, T. Führer, M. Karkulik, M. Melenk, D. Praetorius: Inverse estimates for elliptic integral operators and application to the adaptive coupling of FEM and BEM. in progress.

[2] M. Aurada, M. Feischl, M. Karkulik, D. Praetorius: A posteriori error estimates for the JohnsonNédélec FEM-BEM coupling. Eng. Anal. Bound. Elem.,36 (2012), 255-266.

[3] M. Aurada, S. Ferraz-Leite, D. Praetorius: Estimator reduction and convergence of adaptive BEM. Appl. Numer. Math, in press (2011).

[4] M. Feischl, M. Karkulik, M. Melenk, D. Praetorius: Quasi-optimal convergence rate for an adaptive boundary element method. submitted to SIAM J. Numer. Anal. 


\title{
IOS SUPPORTED Visualisation AND CONTROL OF A SMART-HOME IN THE CONTEXT OF AAL - TECHNOLOGIES
}

\author{
M. Gasser \\ AIT Austrian Institute of Technology GmbH, Austria \\ Corresponding author: M. Gasser, AIT Austrian Institute of Technology GmbH, Health \& Environment \\ Department 2700 Wr. Neustadt, Viktor Kaplan Straße 2, Austria, tcse@matthiasgasser .com
}

Introduction. There is a distinct trend toward an aged society living in single person households. This creates an increasing demand for new support, guidance and assistance services especially for the elderly. Ambient Assisted Living (AAL)[1] copes with this social trend, by embracing technologies which provide various functionality to support people in carrying out daily activities and to provide monitoring for medical reasons. Dwellings equipped with several types of sensors and actuators are usually called Smart-Homes.

HOMER. (HOMe Event Recognition System) is an open and flexible OSGi-based software platform, which aims at the integration of various automation system and consequential event and situation recognition for smart home, addressing comfort, energy efficiency, etc, and Ambient Assisted Living applications, addressing safety, autonomy, self-confidence, etc.[2]

The iOS-Device enabled user interface widens the range of possible Ambient Assisted Living applications tremendously. Utilizing a mobile device for monitoring and controlling one or more Smart-Homes has many advantages over statically installed control terminals, in terms of costs and flexibility. There are mobile driven solutions available, but without any AAL focus.

Implementation. The implementation consists of several components, having an data encoder service using JSON which delivers the the data to a REST - Server module, which is ultimately called by the iOS-Device. Whereby all backend components are written in Java as flexible OSGi bundles, the interface is implemented in Objective-C.

Scenarios. A possible scenario may be an elderly person in care of own relatives. With an AAL equipped home the caregiver will immediately notified, by SMS or push notification, in case of an incident, like an activation of a fall sensor. The mobile user interface would allow a more powerful way of supporting the cared person, by showing more details about latest movements and other activations.

In a clinical or home for the aged context it would allow to optimize therapy work flows based on sensor data feedback, comparing the active - like walking around, ergometer practice, etc — versus the inactive - sleeping, watching television, etc - time.

The interface is also evaluated for use by the people who live in a Smart-Home to control their appliances, like temperature settings, open / close the blinds, and reminders in case of e.g. a forgotten dish in the oven, ready for consumption.

Outlook. The current development snapshot gives already full view over sensor data, (including movements, charting, etc.) and allows control of actuators (switches, dimmer, etc.). Ways to evaluate a current state using Hidden Markov Models are being evaluated. This thesis is still work in progress.

[1] Birgid Eberhardt Reiner Wichert. Ambient assisted living. In Springer, editor, 4. AAL - Kongress 2011, 2011.

[2] HOMER Project AIT. http://homer.aaloa.org/redmine/projects/homer-core 02/2012. 


\title{
Simulation of Pressure Driven Blood Flow in Vessels Defined BY CENTERLINES AND RADIUS PROFILES
}

\author{
C. Godenschwager ${ }^{1,2}$, D. Bernhardt ${ }^{2}, \mathrm{U}$ Rüde $^{1}$ \\ ${ }^{1}$ Friedrich-Alexander-Universität Erlangen-Nürnberg \\ ${ }^{2}$ Siemens Healthcare, Computed Tomography \\ Corresponding author: C. Godenschwager, Friedrich-Alexander-Universität Erlangen-Nürnberg, Chair for System \\ Simulation \\ Cauerstraße 6, 91058 Erlangen, Germany, christian.godenschwager@informatik . uni-erlangen.de
}

Lesions in blood vessels are often examined by medical imaging. Analysis of the hemodynamical impact of such lesions requires a lot of experience of the diagnosing radiologist. The use of simulation tools provides an opportunity to improve accuracy of the diagnosis.

In many cases vascular geometries are given by their centerline and a profile curve, describing the vessels radius at each centerline point. Those can either be the result of an imaging algorithm or an arbitrary input conveniently defining a vessel phantom for the purpose of simulation experiments. To perform a numerical simulation of blood flow in the given vessels a computational domain needs to be created. To apply the Lattice-Boltzmann method, the generation of a three-dimensional cartesian grid with a given cell size is mandatory. Within this grid the cells inside the vascular geometry have to be marked as cells containing a liquid and in the cells surrounding the vessel appropriate boundary conditions have to be set up.

To generate such a cartesian grid we create a set of shapes defined implicitly by signed distance functions from the centerline and radius information. To decide whether a cell is considered inside or outside of the vascular geometry we evaluate the signed distance functions of the shapes. Since only radius information is available we assume a circular cross sections of the vessels. To ensure that the radius specification is adhered to at all times we apply truncated cones to model the space between two centerline points and horn torus segments to model links between them. The cells marking the boundary conditions for in- and outflow are determined by performing morphological dilatation operations at the open ends of the vascular structure. The remaining cells are considered to be obstacles for the blood flow.

Once the cartesian grid containing the vascualar geometry has been created the blood flow simulation is performed using waLBerla (widely applicable Lattice Boltzmann solver from Erlangen, [1]). Especially when simulating blood flow problems it is desirable to close the system at the in- and outflow borders by applying pressure boundary conditions. To achieve this the pressure anti-bounce-back boundary condition scheme [2] was implemented. It was validated using Hagen-Poiseuille flows in pipes of different length, radius, orientation and with different grid resolutions.

[1] C. Feichtinger, S. Donath, H. Köstler, J. Götz, U. Rüde: WaLBerla: HPC Software Design for Computational Engineering Simulations. In: Journal of Computational Science, 2(2): S. 105-112, 2011

[2] I. Ginzburg, F. Verhaeghe, D. d'Humières: Two-relaxation-time lattice Boltzmann scheme: About parametrization, velocity, pressure and mixed boundary conditions. In: Communications in Computational Physics 3, 427, 2008 


\title{
CONVERGENT GEOMETRIC INTEGRATOR FOR THE LANDAU-LIFSHITZ-GILBERT EQUATION WITH MAGNETOSTRICTION
}

\author{
P. GOLDENITS, M. PAGE, D. PRAETORIUS, AND D. SUESS
}

The understanding and development of magnetic materials and devices is of utter relevance for example in magnetic sensors, recording heads, and magneto-resistive storage devices. In the literature it is wellaccepted that dynamic micromagnetic phenomena are described best by the Landau-Lifshitz-Gilbert equation (LLG). This non-linear parabolic equation reads

$$
\begin{aligned}
\mathbf{m}_{t} & =-\alpha \mathbf{m} \times(\mathbf{m} \times \mathbf{H}(\mathbf{m}))+\mathbf{m} \times \mathbf{H}(\mathbf{m}) \\
\mathbf{m}(0) & =\mathbf{m}_{0} \quad \text { in } H^{1}\left(\Omega ; \mathbb{S}^{2}\right) \\
\partial_{n} \mathbf{m} & =0 \quad \text { on }(0, \tau) \times \partial \Omega \\
|\mathbf{m}| & =1 \quad \text { a.e. in }(0, \tau) \times \Omega,
\end{aligned}
$$

where $\mathbf{H}(\mathbf{m})$ denotes the total magnetic field which is given by

$$
\mathbf{H}(\mathbf{m}):=\Delta \mathbf{m}+D \Phi(\mathbf{m})+\mathcal{P}(\mathbf{m})-\mathbf{f}+\mathbf{h}^{\boldsymbol{\sigma}}(\mathbf{m})
$$

and consists of the exchange field, the anisotropy field, the stray-field, the applied field, as well as the contribution of the non-linear magnetostrictive field. In this equation, the magnetic behavior of the ferromagnetic body is characterized by the vector valued magnetization $\mathbf{m}:(0, \tau) \times \Omega \rightarrow \mathbb{R}^{3}$. In addition, the magnetostrictive field $\mathbf{h}^{\boldsymbol{\sigma}}(\mathbf{m})$ depends on the so-called stress tensor $\boldsymbol{\sigma}$ and is thus coupled to the equation of elastodynamics

$$
\varrho \mathbf{u}_{t t}-\nabla \cdot \boldsymbol{\sigma}=0 \quad \text { on }(0, \tau) \times \Omega .
$$

Numerical challenges for the time integration arise from the strong non-linearity, the non-convex side constraint $|\mathbf{m}|=1$, the non-local dependence of the demagnetization field $\mathcal{P}(\mathbf{m})$ from the magnetization $\mathbf{m}$ as well as from the coupling of the two equations.

The great number of applications as well as the amount of numerical issues makes LLG of equal interest for both, physicists and mathematicians, and thus the scientific community benefits in many ways.

Recently there has been a huge progress in the mathematical literature for the so-called small-particle limit. In this model all energy terms but the exchange energy are neglected, i.e. the magnetic field is simplified to $\mathbf{H}(\mathbf{m})=\Delta \mathbf{m}$, cf. [Alouges/Jaisson, Math. Models Methods Appl. Sci., 16 (2006)], [Bartels/Prohl, SIAM J. Numer. Anal., 44 (2006)], and [Alouges, Discrete Contin. Dyn. Syst. Ser. S, 1 (2008)].

In our contribution, we generalize the approach of ALOUGES to the total magnetic field stated above, i.e. including all five energy terms and combine it with the approach from BANAS/SLODICKA, cf. [Banas/SlodickA, Appl. Numer. Math., 56 (2006)] for the discretization of the second equation. Since the computation of the demagnetization field is very time and memory consuming, the proposed time integrator is split into an implicit part and an explicit part. The first one deals with the higher-order term $\Delta \mathbf{m}$ stemming from the exchange energy, whereas all other terms are treated explicitly. In addition, the two equations can be decoupled. As the original algorithm, our extension guarantees the side constraint $|\mathbf{m}(t, \mathbf{x})|=1$ for all nodes $\mathbf{x}$ and all time-steps $t$ to be fulfilled as well as unconditional convergence if spatial mesh-size $h$ and time-step size $k$ tend to zero. In contrast to previous works, another benefit of our scheme is the fact that it requires only to solve two linear systems per time-step. Finally, our analysis allows to replace the operator $\mathcal{P}$ which maps $\mathbf{m}$ onto the corresponding demagnetization field, by a discrete operator $\mathcal{P}_{h}$. Possible choices for $\mathcal{P}_{h}$ are given by an extended convolution operator, the FEM-BEM coupling, or the hybrid FEM-BEM approach proposed in [FREDKIN/KoEHLER, IEEE Transactions on Magnetics 26 (1990)] which is mostly used in the Physics literature. 


\title{
Vascular ImPedance Calculation Using A New Blood Flow Model
}

\author{
B. Hametner ${ }^{1,2}$, S. Wassertheurer ${ }^{1,2}$, T. Weber ${ }^{3}$ \\ ${ }^{1}$ AIT Austrian Institute of Technology GmbH, Austria \\ ${ }^{2}$ Vienna University of Technology, Austria \\ ${ }^{3}$ Klinikum Wels-Grieskirchen, Austria
}

Corresponding author: B. Hametner, AIT Austrian Institute of Technology GmbH, Health \& Environment
Department, Muthgasse 11, 1190 Vienna, Austria, bernhard.hametner. fl@ait.ac.at

Introduction. Cardiovascular diseases are the major cause of death in many developed countries, and the World Health Organization (WHO) predicts a worldwide increase for the next centuries. Within the concept of pulse wave analysis, arterial pressure and flow curves over a whole cardiac cycle are analyzed. A possibility to characterize the arterial system is to relate pressure and flow via a linear time-invariant transfer function. The transfer function can be expressed as the ratio of output to input in the frequency domain. This ratio of pressure to flow is called impedance. The characteristic impedance $\left(Z_{c}\right)$ is obtained when pressure and flow waves are not influenced by wave reflection. This situation will never occur in the arterial tree, therefore the characteristic impedance can be approximated only, usually with the help of a calculated input impedance.

Methods. Since the measurement of blood flow in the aorta is cumbersome, models are used to generate flow curves for the determination of vascular impedance. The aim of this work is to evaluate the effects of a new blood flow model on the determination of the characteristic impedance compared to flow curves gained from ultrasound measurements. This recently developed blood flow model is based on Windkessel theory (ARCSolver flow). By optimizing the left ventricular work using the calculus of variations a personalized flow curve can be obtained.

In a study population of 148 patients, pressure and flow curves were measured non-invasively using tonometric and ultrasound techniques. For the evaluation of the different models the input impedance and subsequently the characteristic impedance will be calculated in the frequency domain. For a fair comparison for both methods the flow curves are scaled in such a way that the maximal value is at the level of 100 arbitrary units (AU).

Results. The mean characteristic impedance using flow curves from ultrasound images is 0.22 (0.08 SD) AU. For the ARCSolver flow a mean difference of 0.016 (0.039 SD) AU compared to the ultrasound flow is obtained. The correlation between the two methods is $\mathrm{R}=0.88$. In a Bland-Altman analysis small trends of underestimation for higher values can be noticed, see the figure below for a scatter plot and a Bland-Altman plot.
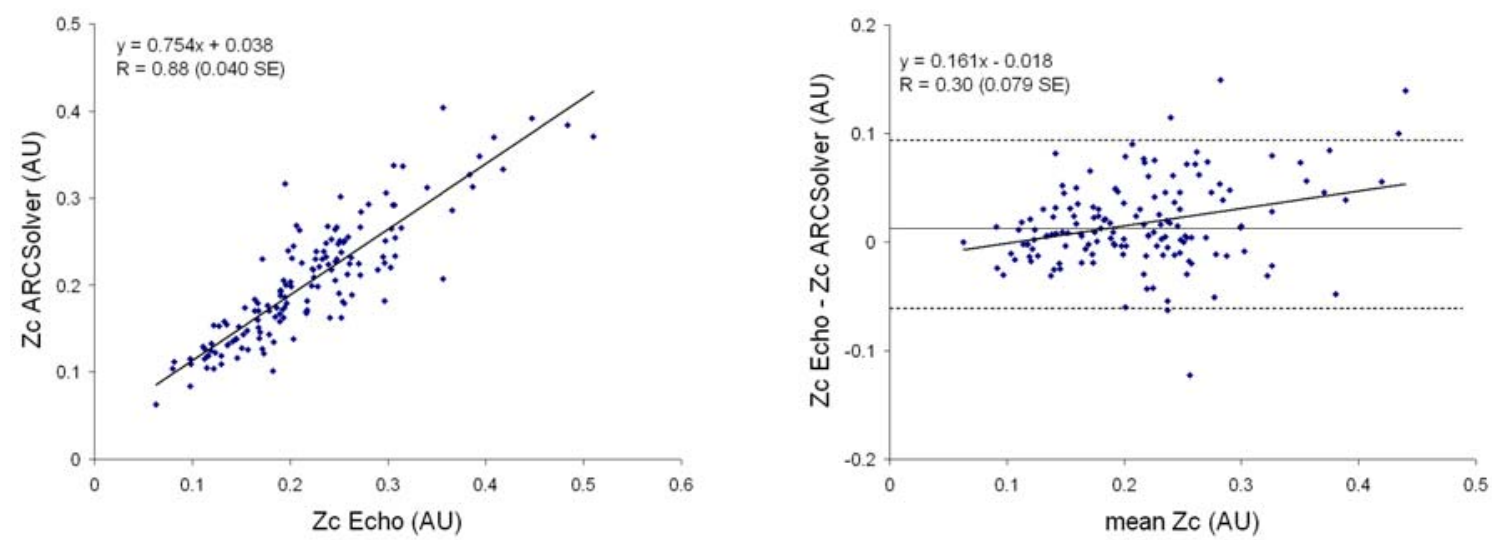

Scatter plot (left) and Bland-Altman plot (right) of the characteristic impedance obtained with flow from ultrasound images (Echo) and from a blood flow model (ARCSolver)

Conclusion. These results indicate that calculations using the ARCSolver flow model provide good estimates for characteristic impedance. Since these impedance values have a sufficient accuracy compared to those gained from ultrasound measurements, this new model offers an easy way to perform pulse wave separation and furthermore to calculate pulse wave velocity based on information from a single blood pressure measurement, where characteristic impedance is an important parameter for the calculations.

[1] Nichols W. and O'Rourke M.: McDonald's Blood Flow in Arteries. Arnold, London, 4th edition, 1998.

[2] Weber T., Haiden A., Hametner B., Mayer C., Kropf J., Wassertheurer S., and Eber B.: Aortic pulse wave velocity, estimated with a simplified method based on radial waveforms and body height, predicts cardiovascular events. Artery Research. 2011;5:178. 


\title{
High-Level Modelling And Simulation of Machine ToOls in ENERGY OPTIMIZATION OF PRODUCTION PLANTS
}

\author{
B. Heinzl, Vienna University of Technology, Austria; \\ N. Popper, M. Landsiedl, dwh Simulation Services, Austria; \\ Ch. Dorn, S. Brandstetter, A. Dimitriou, Vienna University of Technology, Austria \\ Corresponding author: B. Heinzl, Vienna University of Technology, \\ Institute for Analysis and Scientific Computing, \\ 1040 Wien, Wiedner Hauptstraße 8-10, Austria, bernhard . heinzl@tuwien . ac . at
}

Introduction. The research project INFO - as one of several current research projects - aims at increasing energy efficiency in production plants. In this project, this is done by considering various disciplines of energy technology, production technology and building design in a holistic approach [1]. By creating a comprehensive simulation model including all micro- and macro-structures, it attempts to make qualified predictions about the effectiveness of different energy saving approaches and identify potential savings in manufacturing plants [2]. In contrast to previous (rather static) concepts, this approach allows customized recommendations based on models of the real facility.

One step of this project includes energy considerations and development of simulation models of the microstructures of production plants (individual processes and machines) in order to point out optimization potential and gain knowledge for merging the individual levels in an ultimately complete simulation. Some of these aspects are studied in more detail by creating a multi-domain model of a turning lathe as an example of a machine tool.

Modelling Method. By using a high-level object-oriented component-based modelling method (also called Physical Modelling), we can combine electrical, mechanical as well as thermal aspects of a machine tool in a structural manner. The underlying concept of reusing components of modularly structured models also allows for an easy adaptation of certain parts of the model for other machines later. In order to be able to validate the developed model against real measurement data, we attempt to create a model of an actual turning lathe, which is provided by the Institute for Production Engineering and Laser Technology from the Vienna University of Technology.

Although simulators for object-oriented component-based modelling of physical systems have evolved considerably in the last years, there are still numerical issues when it comes to simulating complex multi-domain systems. Developing our model in several stages with gradually increasing level of detail lets us identify the numerical boundaries of the simulation as well as the degree of modelling effort necessary for considering certain reasonable scenarios, therefore combining the bottom-up modelling approach of the component-based concept with top-down modelling.

Modelling Stages. The development of the simulation model is done in three stages. The first stage contains the main mechanical and electrical components like electric drives, power supply, gear belt drive, leadscrew, slides for automatic feed and mechanical loads. It also includes a simple calculation model of the cutting forces using common formulas and parameters [3]. In the second step, the model is extended by the motor control for all drive motors and thermal components, which take the generated waste heat into account. However, since the second stage leads us to some numerical boundaries, some adjustments have to be included in the model in order to enable appropriate simulation performance. The simulation model in the last stage will furthermore include remaining electrical loads and additional details like elasticity of mechanical parts or heating of the workpiece.

Conclusion. The object-oriented Physical Modelling approach seems to be a suitable tool for multi-domain modelling of machine tools. The big advantage of creating modular, easily refinable and modifiable models has been proven. However, appropriate parameterization of our simulation model turns out to have been underestimated, since many of the needed parameters are not shown in data sheets and their identification would require extensive measurements on the machine tool.

Future work will focus on refinement of the models as part of the next stages in the top-down modelling process as well as the parameterization of the remaining components. For model validation it is planned to compare the simulation results of various scenarios against measurement data obtained from the turning lathe.

[1] Dorn Ch. and Bleicher F.: Interdisziplinäre Forschung zur Energieoptimierung in Fertigungsbetrieben. Junior Science Conference 2010.

[2] http://www.projekt-info.org

[3] Degner W., Lutze H. and Smejkal E.: Spanende Formung, Theorie - Berechnung - Richtwerte. Hanser Verlag, 2009. 


\title{
GPU-ACCELERATED MRI-SiMULATION AND VISUALIZATION ON A CAVE-LIKE ENVIRONMENT
}

\author{
M. Georgiev, D. Gudu, M. Hinterstocker, T. Kovačević, A. Shahvarani \\ Technische Universität München, Germany \\ Corresponding author: T. Kovačević, tijana.kovacevic@in.tum.de
}

Introduction. While magnetic resonance imaging (MRI) is based on well-understood principles, building an actual machine presents numerous difficulties, including expense. For this reason, simulating an MRI's behavior can be very useful. MRI simulations have proven to provide a powerful means in the respective development and research processes. We present a portable tool for parallel MRI simulation and visualization of the results on a multi-monitor system.

Implementation. In order to achieve portability, the application was written using cross-platform libraries, including Qt, CUDA, OpenGL and Equalizer, which can be compiled for both Windows and Linux. A graphical user interface allows the user to specify problem-specific parameters and the body model of interest. The GPUaccelerated simulator is written in $\mathrm{C}++$ with CUDA and uses the isochromat summation method. When given a virtual three-dimensional phantom, the simulation processes it on a slice-by-slice basis. The simulation results can be compared against the input phantom. The results can be visualized either via volume rendering or on a slice-by-slice basis. In either case, the visualization uses OpenGL.

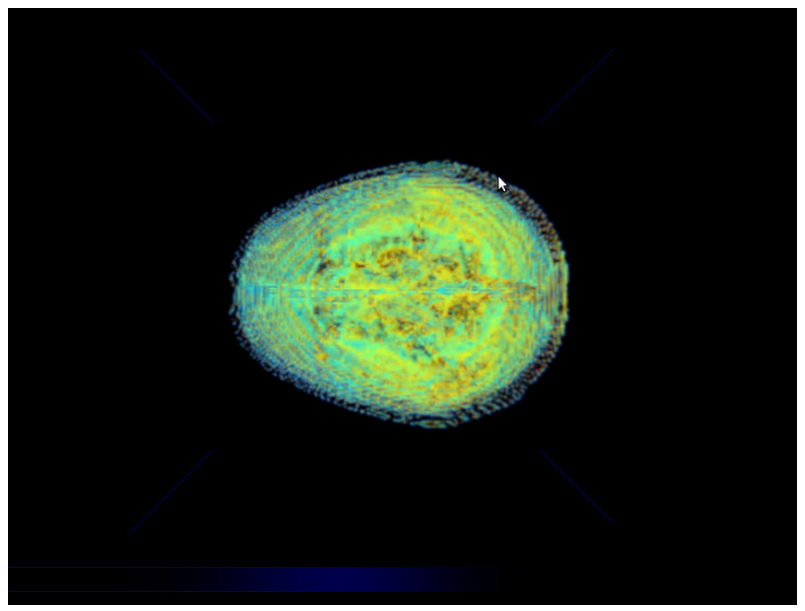

3D visualization of an MRI dataset

Results. We present performance results of the parallel implementation compared to a serial version and a detailed description of the simulation environment.

[1] H. Benoitcattin, G. Collewet, B. Belaroussi, H. Saintjalmes, and C. Odet. The SIMRI project: a versatile and interactive MRI simulator. Journal of Magnetic Resonance, 173(1):97-115, March 2005.

[2] FRAVE: flexible virtual reality system. http://www.physorg.com/news/2011-05-frave-flexible-virtualreality.html. 


\title{
STUDIES ON THE THERMODYNAMICAL BEHAVIOUR OF A MACHINE TOOL AND ITS ENVIRONMENT
}

\author{
Matthias Rößler, Vienna University of Technology, Austria \\ Corresponding author: Matthias Rößler \\ ARGESIM Masterkolleg \\ Vienna University of Technology - 1040 Wien, Wiedner Hauptstraße 8-10 - Austria \\ Email: matthias.roessler@tuwien.ac.at
}

Introduction Energy optimization is a very fascinating topic. For this reason, the research project INFO ${ }^{1}$, which is supported by the FFG, the Austrian Research Promotion Agency, tries to make a comprehensive simulation of a production plant, including micro- and macroscopic aspects, like machines, processes, the building envelope and so on, to be able to make qualified predictions about the impact of certain energy-saving measures. In this context, the issue of coupling different model-parts is of very high interest. This work focuses on two parts of the simulation, a simple machine tool, namely a linear guiding device, and the environment surrounding it. The chosen modelling approach is physical modelling, a block-based, acausal and object-oriented approach to model physical systems. The Modelica Corp. provides a standard to describe physical systems and also provides a standard-library, where common blocks are defined. By choosing the same approach for both model parts, the coupling of the two will be less difficult than having to merge completely different models.

Machine tool The linear guiding device is a very simple part of the machine tool. It consists of a permanent magnet DC motor that is connected to a thread bar via a gear belt. The thread bar moves a cart, where the sliding mass is attached. For the coupling the dissipative elements of the system have to be considered. In this case, these are the electric motor, the bearing friction and the friction between the thread bar and the cart. The heat emmission of these components is calculated in the respective blocks and can then be used as a heating source for the environment model. For the validation of this partial model there is an actual test setup, where measurements can be taken.

Environment For the model of the environment a compartment-model is chosen. There are several assumptions made for the model:

- Each compartment has its own thermal mass, which depends on its volume and the specific heat capacity of the air

- For the heat transfer into adjacent compartments only thermal conductance is considered

- The thermal properties of the air are assumed to be constant over the simulation run.

- The walls of the room are assumed to be perfectly isolated, so there is no heat exchange between the compartments and the adjacent walls and therefor no energy is lost in the system.

Under those assumptions a rather easy model of the environment can be made. The advantage of this model is, that it can easily be refined if a higher resolution should be necessary.

For the coupling of the two partial models the heat emission of the dissipative parts of the machine tool can easily

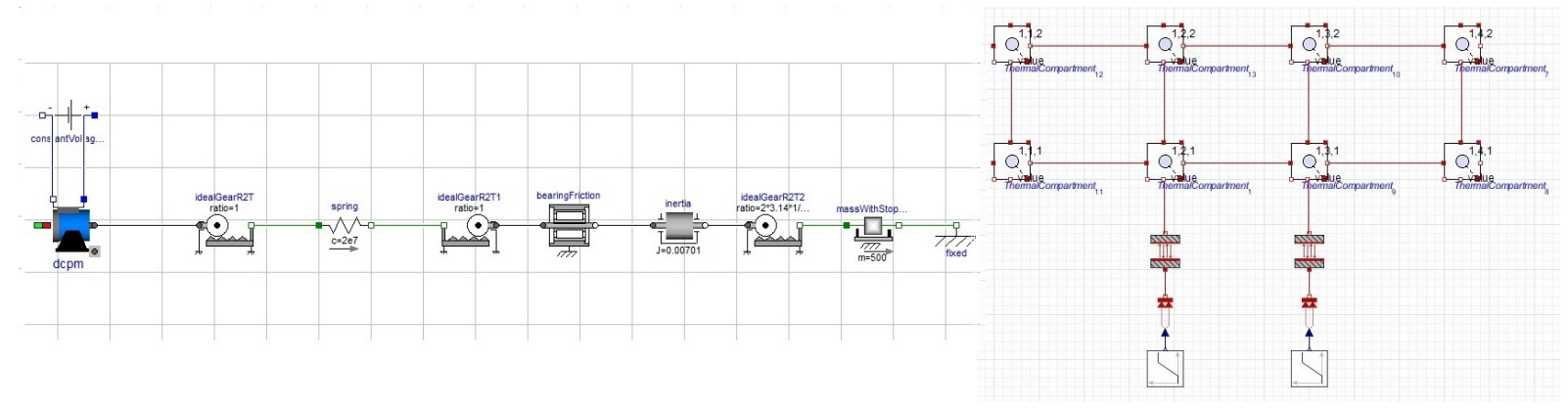

Figure 1: Model of the linear guiding device on the left and of the environment on the right

be considered the heat sources of the environment-model.

[1] Modelica Corporation, Modelica - A Unified Object-Oriented Language for Physical Systems Modeling Language Specification Version 3.2, March 24, 2010

https : //www.modelica.org/documents/ModelicaSpec32.pdf

${ }^{1}$ http://www.projekt-info.org 


\title{
Using Open Source Geo-Data in Agent-Based Models of Health CARE UTILIZATION
}

\author{
G. Romstorfer, G. Schneckenreither \\ Vienna University of Technology, Austria; dwh simulation services, Vienna, Austria \\ Corresponding author: G. Romstorfer, \\ Vienna University of Technology, Institute for Analysis and Scientific Computing \\ 1040 Wien, Wiedner Hauptstraße 8-10, Austria, georg . romstorfer@gmail .com
}

\begin{abstract}
Introduction. The process of choosing medical providers for consuming health care services depends on a variety of properties. Among them are the type of required service, coverage of health insurance, quality of the facility, accumulation of multiple treatment options and the distance to the patients domicile. In a nation/system wide dynamic simulation distance becomes a fundamental property that confronts us with different problems. We discuss the integration of geographic information into an agent-based model for simulating the dynamic distribution of patient-provider relations based on real health care data. Our work is connected to a research cooperation of Vienna University of Technology and the Main Association of Austrian Social Security Institutions.
\end{abstract}

Geographic Information. To succeed in processing geographic data a common coordinate reference system (CRS) and tools like software libraries and database systems which are capable of performing geographic transformations, calculations or queries are of high importance. Projects like QuantumGIS, PostGIS and OpenStreetMap (OSM), which are developed by the Open-source community, have proven to be the best choice for our purposes. They provide a tool-chain for organising and processing geographic data that is free of cost, of high quality, state of the art and also allows us to use a huge database of cartographic data (OSM) as well as combine data from different sources (e.g. Statistics Austria) and of different type (e.g. raster data).

The most challenging part of obtaining and preprocessing geographic information lies in bringing health care data into a geographic context. Especially this data is very sensitive in terms of protection of privacy and thus must be anonymised, clustered or even blurred. A very direct consequence of these processes is that identification of single persons and their history of visited providers (i.e. their decisions) is no longer possible. This fact is unavoidable but does clearly not render any dynamic simulation based on anonymised data useless in the first place [1].

An even greater challenge are incomplete data sets, inconsistent naming systems or complete lack of information enabling geographic allocation. The reason for these drawbacks are the great number of different institutions involved in the processes of initial data acquisition and the lack of an immanent necessity to collect geographic data. Furthermore reverse techniques have to be used to regenerate a reasonable distribution of the locations of the patients' domiciles. This can be achieved by randomly distributing the domiciles based on the actual population density and the geographic information included in the health care data.

Techniques. Integration of geographic information into an agent-based model requires several steps:

- Combining all required data into a single database. Preceding this decompression and extraction of data from different sources and formats, alignment of nomenclature and transformation to a common CRS is necessary.

- Geographic locations (coordinates) must be assigned to all agents (providers and patients) based on addresses, postcodes or political structures. This can be achieved by searching cartographic data (OSM) or by the technique mentioned at the end of the last section. Latter is based on raster data on population count which must be combined with a political structuring. As a consequence we have to solve a large number of point-in-polygon problems [2] in order to distribute the patients in the resulting polygon grid (intersections of raster cells and political districts).

- Since simulation speed is also an issue, computationally intensive operations like finding the shortest path between two locations or deciding whether a location lies within a certain area must be precalculated.

- Integration of the resulting database into a dynamic simulation requires program libraries or modelling frameworks which are capable of accessing database systems, provide basic geographic processing tools and allow appropriate (even interactive) graphic display of results.

Tasks 1-3 can be combined in an automated preprocessing tool, which allows to quickly react on the highly dynamic geographic data available from OSM. The implementation of our test model relies on AnyLogic, GeoTools and self-written Java classes.

[1] Urach C., Breitenecker F., Miksch F., Popper N., Wilbacher I., Zauner G.: Distance Dependence on the Willingness of Patients to Participate in Ambulatory Heart Rehabilitation Programs. SMDM Society for medical Decision Making Europe 2010 - Public Health Decision Making, Target Conferences Ltd., 2010.

[2] Haines E.: Point in Polygon Strategies. Graphics Gems IV, (Ed.: Paul Heckbert), Academic Press, 1994, 24-46. 


\section{Participants of TCSE Workshop Vienna 2012}

\begin{tabular}{|c|c|c|c|c|}
\hline Surname & $\begin{array}{l}\text { First } \\
\text { Name }\end{array}$ & email & Institution & State \\
\hline Albertini & Daniel & e0402305@student.tuwien.ac.at & $\begin{array}{l}\text { Vienna University of } \\
\text { Technology, Institute of Analysis } \\
\text { a. Scientific Computing }\end{array}$ & Austria \\
\hline Alkilani & Salah & e0327022@sstudent.tuwien.ac.at & $\begin{array}{l}\text { Vienna University of } \\
\text { Technology, Institute of Analysis } \\
\text { a. Scientific Computing }\end{array}$ & Austria \\
\hline Amini & Ahmad & Ahmad.Amini@tuwien.ac.at & $\begin{array}{l}\text { Vienna University of } \\
\text { Technology, Institute of Analysis } \\
\text { and Scientific Computing }\end{array}$ & Austria \\
\hline Anderl & Daniela & daniela.anderl@Istm.uni-erlangen.de & $\begin{array}{l}\text { University of Erlangen, Institute } \\
\text { for Fluid Mechanics }\end{array}$ & Germany \\
\hline Bachler & Martin & martin.bachler.fl@ait.ac.at & $\begin{array}{l}\text { Vienna University of } \\
\text { Technology, Institute of Analysis } \\
\text { a. Scientific Computing }\end{array}$ & Austria \\
\hline Bauer & Andreas & andreas.e101.bauer@tuwien.ac.at & $\begin{array}{l}\text { Vienna University of } \\
\text { Technology, Institute of Analysis } \\
\text { a. Scientific Computing }\end{array}$ & Austria \\
\hline Baumgartner & Stefan & stefan.baumgartner@univie.ac.at & University of Vienna & Austria \\
\hline Bicher & Martin & martin.bicher@tuwien.ac.at & $\begin{array}{l}\text { Vienna University of } \\
\text { Technology, Institute of Analysis } \\
\text { a. Scientific Computing }\end{array}$ & Austria \\
\hline Breitenecker & Katharina & katharina.breitenecker@tuwien.ac.at & $\begin{array}{l}\text { International Atomic Energy } \\
\text { Agency }\end{array}$ & Austria \\
\hline Breitenecker & Felix & felix.breitenecker@tuwien.ac.at & $\begin{array}{l}\text { Vienna University of } \\
\text { Technology, Institute of Analysis } \\
\text { a. Scientific Computing }\end{array}$ & Austria \\
\hline Bruckner & Martin & martin.bruckner@tuwien.ac.at & $\begin{array}{l}\text { Vienna University of } \\
\text { Technology, Institute of Analysis } \\
\text { a. Scientific Computing }\end{array}$ & Austria \\
\hline Descovich & Xenia & xenia.descovich@tuwien.ac.at & $\begin{array}{l}\text { Vienna University of } \\
\text { Technology, Institute of Analysis } \\
\text { and Scientific Computing }\end{array}$ & Austria \\
\hline
\end{tabular}




\begin{tabular}{|c|c|c|c|c|}
\hline Ecker & Horst & horst.ecker@tuwien.ac.at & $\begin{array}{l}\text { Vienna University of } \\
\text { Technology, Inst. of Mechanics } \\
\text { and Mechatronics }\end{array}$ & Austria \\
\hline Ecker & Brigitte & brigitte.ecker@tuwien.ac.at & $\begin{array}{l}\text { Vienna University of } \\
\text { Technology, Institute of Analysis } \\
\text { a. Scientific Computing }\end{array}$ & Austria \\
\hline Eckhardt & Wolfgang & eckhardw@in.tum.de & $\begin{array}{l}\text { TU München, Institut für } \\
\text { Informatik } 5\end{array}$ & Germany \\
\hline Einzinger & Patrick & patrick.einzinger@drahtwarenhandlung.at & DWH Simulation Services & Austria \\
\hline Englberger & Julian & julian.englberger@googlemail.com & UAS Regensburg / IHI Zittau & Germany \\
\hline Erhart & Jacqueline & jerhart@ati.ac.at & $\begin{array}{l}\text { Vienna University of } \\
\text { Technology, Inst. of Atomic and } \\
\text { Subatomic Physics }\end{array}$ & Austria \\
\hline Fitsch & Ursula & ursula.fitsch@tuwien.ac.at & $\begin{array}{l}\text { Vienna University of } \\
\text { Technology, Institute of Analysis } \\
\text { a. Scientific Computing }\end{array}$ & Austria \\
\hline Führer & Thomas & thomas.fuehrer@tuwien.ac.at & $\begin{array}{l}\text { Vienna University of } \\
\text { Technology, Institute of } \\
\text { Analysis a. Scientific Computing }\end{array}$ & Austria \\
\hline Gasser & Matthias & e0402792@student.tuwien.ac.at & $\begin{array}{l}\text { Vienna University of } \\
\text { Technology, Institute of Analysis } \\
\text { a. Scientific Computing }\end{array}$ & Austria \\
\hline Glock & Barbara & e0225107@student.tuwien.ac.at & DWH Simulation Services & Austria \\
\hline Godenschwager & Christian & $\begin{array}{l}\text { christian.godenschwager@informatik.uni- } \\
\underline{\text { erlangen.de }}\end{array}$ & $\begin{array}{l}\text { Friedrich-Alexander-Universität } \\
\text { Erlangen-Nürnberg }\end{array}$ & Germany \\
\hline Goldenits & Petra & petra.goldenits@tuwien.ac.at & $\begin{array}{l}\text { Vienna University of } \\
\text { Technology, Institute of } \\
\text { Analysis a. Scientific Computing }\end{array}$ & Austria \\
\hline Hafner & Irene & irene.hafner@tuwien.ac.at & $\begin{array}{l}\text { Vienna University of } \\
\text { Technology, Institute of Analysis } \\
\text { a. Scientific Computing }\end{array}$ & Austria \\
\hline Hametner & Bernhard & bernhard.hametner@tuwien.ac.at & $\begin{array}{l}\text { Vienna University of } \\
\text { Technology, Institute of Analysis } \\
\text { and Scientific Computing }\end{array}$ & Austria \\
\hline
\end{tabular}




\begin{tabular}{|c|c|c|c|c|}
\hline Haumer & Anton & Anton.Haumer@ait.ac.at & Austrian Institute of Technology & Austria \\
\hline Heinzl & Bernhard & bernhard.heinzl@tuwien.ac.at & $\begin{array}{l}\text { Vienna University of } \\
\text { Technology, Institute of Analysis } \\
\text { and Scientific Computing }\end{array}$ & Austria \\
\hline Hinterstocker & Michael & michael.hinterstocker@mytum.de & $\begin{array}{l}\text { TU München, Institut für } \\
\text { Informatik - Lehrstuhl I5 (SCCS) }\end{array}$ & Germany \\
\hline Hoellrigl-Binder & Sonja & sonja.hoellrigl-binder@tuwien.ac.at & $\begin{array}{l}\text { Vienna University of } \\
\text { Technology, Institute of Analysis } \\
\text { and Scientific Computing }\end{array}$ & Austria \\
\hline Hollaus & Karl & karl.hollaus@tuwien.ac.at & $\begin{array}{l}\text { Vienna University of } \\
\text { Technology, Institute of Analysis } \\
\text { and Scientific Computing }\end{array}$ & Austria \\
\hline Hrabal & David & davic.hrabal@uni-ulm.de & Ulm University & Germany \\
\hline Kemmetmüller & Wolfgang & wolfgang.kemmetmueller@tuwien.ac.at & $\begin{array}{l}\text { Vienna Universitty of } \\
\text { Technology Institute of } \\
\text { Automation and Control }\end{array}$ & Austria \\
\hline Konrad & Gertrud & gkonrad@ati.ac.at & $\begin{array}{l}\text { Vienna University of } \\
\text { Technology, Inst. of Atomic and } \\
\text { Subatomic Physics }\end{array}$ & Austria \\
\hline Körner & Andreas & akoerner@asc.tuwien.ac.at & $\begin{array}{l}\text { Vienna University of } \\
\text { Technology, Institute of Analysis } \\
\text { a. Scientific Computing }\end{array}$ & Austria \\
\hline Kovacevic & Tijana & tijana.kovachevic@gmail.com & Technische Universität München & Germany \\
\hline Leskovar & Ruth & e0726512@student.tuwien.ac.at & $\begin{array}{l}\text { Vienna University of } \\
\text { Technology, Institute of Analysis } \\
\text { a. Scientific Computing }\end{array}$ & Austria \\
\hline
\end{tabular}


Maurer

Werner

maur@zhaw.ch

Nagele-Wild

Nicole

nicole.nagele@tuwien.ac.at

Nannen

Lothar

lothar.nannen@tuwien.ac.at

Page

Marcus

marcus.page@tuwien.ac.at

Pawletta

Thorste

thorsten.pawletta@hs-wismar.de

Pöll

Carina

carina.poell@tuwien.ac.at

Pontrelli

Giusepp

g.pontrelli@iac.cnr.it

Popper

Nikolas

niki.popper@drahtwarenhandlung.at

DWH Simulation Services

CNR Roma Istituto per le

Applicazioni del Calcolo

Wismar University of Applied Sciences

Germany

Vienna University of

Technology, Institute of Analysis a. Scientific Computing

Italy

Vienna University of

Technology, Institute of Analysis a. Scientific Computing

Austria

Praetorius Dirk dirk.praetorius@tuwien.ac.at

Romstorfer
Austria

Austria
Vienna University of

Technology, Institute of Analysis a. Scientific Computing 
Rößler

Matthias

matthias.roessler@tuwien.ac.at

Rozsenich

Benjamin

benjamin.rozsenich@tuwien.ac.at

Schneckenreiter Günter

gschneck@seth.asc.tuwien.ac.at

Stöhr

Markus

$\underline{\text { markus.stoehr(at)boku.ac.at }}$

Striebel

Michael

michael.striebel@math.uni-wuppertal

Tauböck

Shabnam

shabnam.tauböck@tuwien.ac.at

Christoph

christoph.urach@drahtwarenhandlung.at

Urach

Chistoph

Wastian Matthias matwaste@gmx.at

Wild

Wolfgang

wolfgang.wild@tuwien.ac.at

Stefanie Nadine stefanie.winkler@tuwien.ac.at
DWH Simulation Services

Austria

University of Vienna

Austria

Bergische Universität Wuppertal

Germany

DWH Simulation Services

Vienna University of

a. Scientific Computing

Vienna University of

Technology, Institute of Analysis Austria

a. Scientific Computing

Vienna University of

Technology, Institute of Analysis Austria

a. Scientific Computing

Austria

Vienna University of

Technology, Institute of Analysis

a. Scientific Computing

Austria

Vienna University of

Technology, Institute of Analysis

Austria

a. Scientific Computing

Vienna University of

Technology, Institute of Analysis Austria

a. Scientific Computing 
Winterer

Nina

nina.winterer@gmx.at

Wytrzens

Claudia

e0825785@student.tuwien.ac.at

Zimmermann

Andreas

e0425067@student.tuwien.ac.at

Zechner

Anna

anna.zechner@tuwien.ac.at

Hagendorf

Olaf

olaf.hagendorf@hs-wismar.de

Wiechert

Wolfgang

w.wiechert@fz-juelich.de

dieter.suess@tuwien.ac.at

Fohler

Armin

e0425293@student.tuwien.ac.at
Vienna University of

Technology, Institute of Analysis

Austria

a. Scientific Computing

Vienna University of

Technology, Institute of Analysis

a. Scientific Computing

Austria

Vienna University of

Technology, Institute of Analysis

a. Scientific Computing

Austria

Vienna University of

Technology, Institute of Analysis

Austria

a. Scientific Computing

Wismar University of Applied Sciences

Germany

Forschungszentrum Jülich

Germany

Vienna University of

Technology, Institute of Solid Austria State Physics

Vienna University of

Technology, Institute of Analysis

a. Scientific Computing

Austria

Vienna University of

Technology, Institute of

Computer Engineering
Austria 



\section{ABOUT ASIM}

ASIM (Arbeitsgemeinschaft Simulation) is the association for simulation in the German speaking area. Organisationally, ASIM is a part of the GI - Gesellschaft für Informatik, the German Association for Informatics.

ASIM is organised in Working Groups:

- Methods in Modelling and Simulation

- Simulation in Environmental Sciences, Medicine and Biology

- Simulation of Technical Systems

- Simulation in Production and Logistics

- Simulation of Traffic Systems

- Simulation in / for Education

ASIM is involved in the following international activities:

- Co-Sponsoring / co-organisation of the tri-annual EUROSIM Congress

- Co-organisation of the tri-annual conference series MATHMOD Vienna

- Publication of the journal SNE - Simulation Notes Europe

- Organisation of the TCSE Workshop Series, etc.

\section{ABOUT TCSE WORKSHOP SERIES}

Modelling and simulation of complex systems from many fields of application requires awide spectrum of methods, algorithms, and tools that cannot be surveyed even by experts.

For many problems different solution approaches exist and the choice of a suitable method is usually determined by the modeller's experience and preferences. Direct comparisons of methods in practical applications are not commonly found in literature. The workshop series Trends in Computational Science and Engineering - TCSE - tries to fill this void.

The TCSE workshop is particularly addressing advanced students and PhD candidates and is based on two scientific pillars:

- Overview Talks on trends in CSE

- Poster Session of PhD and Master Thesis projects in CSE and Modelling and Simulation 